\title{
An Effort Towards Full Graphene Photodetectors
}

\author{
Farhad LARKI ${ }^{1 *}$, Yaser ABDI ${ }^{2}$, Parviz KAMELI ${ }^{1}$, and Hadi SALAMATI ${ }^{1}$ \\ ${ }^{1}$ Department of Physics, Isfahan University of Technology, Isfahan 84156-83111, Iran \\ ${ }^{2}$ Nanophysics Research Lab, Department of Physics, University of Tehran, Tehran 84156-83111, Iran \\ *Corresponding author: Farhad LARKIＥ-mail: f.larki@ph.iut.ac.ir
}

\begin{abstract}
Graphene as a truly 2-dimensional (2D) system is a promising candidate material for various optoelectronic applications. Implementing graphene as the main building material in ultra-broadband photodetectors has been the center of extensive research due to its unique absorption spectrum which covers most of the electro-magnetic spectra. However, one of the main challenges facing the wide application of pure graphene photodetectors has been the small optical absorption of monolayer graphene. Although novel designs were proposed to overcome this drawback, they often need complicated fabrication processes in order to integrate with the graphene photodetector. In this regard, fabrication of purely graphene photodetectors is a promising approach towards the manufacturing of simple, inexpensive, and high photosensitive devices. The fabrication of full graphene photodetectors (FGPDs) is mainly based on obtaining an optimal technique for the growth of high quality graphene, modification of electronic and optical properties of the graphene, appropriate techniques for transfer of graphene from the grown substrate to the desire position, and a proper design for photodetection. Therefore, the available states of the art techniques for each step of device fabrication, along with their pros and cons, are reviewed and the possible approaches for optimization of FGPDs have been proposed.
\end{abstract}

Keywords: Graphene; photodetectors; graphene growth; photodetection

Citation: Farhad LARKI, Yaser ABDI, Parviz KAMELI, and Hadi SALAMATI, "An Effort Towards Full Graphene Photodetectors," Photonic Sensors, 2022, 12(1): 31-67.

\section{Introduction}

As an exotic 2-dimansional (2D) material, graphene is an attractive material for future optoelectronic devices due to its excellent electronic and optical properties, such as variety of transport phenomena that are characteristics of 2D Dirac fermions, near-ballistic transport at room temperature, bipolarity, high purity, high mobility, high critical current density, linear dispersion of the Dirac electrons, and saturable absorption [1-7]. In addition to many potential applications of grapheme, such as touch screen displays [8], flexible electronic devices [9], organic light-emitting diodes (OLEDs) [10], high-frequency transistors [11], tissue engineering applications, regenerative medicine [12], optical modulator [13], mode-locked laser/THz generator [14], and solar cells $[15,16]$, graphene can also be used as a promising material for ultra-broadband photodetectors. For implementation of graphene in any of the above applications, it is crucial to know the fundamental properties of the graphene as well as suitable techniques for fabrication of the graphene based devices. Therefore, in this work we have tried to provide a comprehensive study on the prerequisites for 
fabrication of full graphene photodetectors (FGPDs) from the perspectives of graphene growth methods, the transfer techniques of graphene, photodetection mechanism in graphene based devices, and the modifications that have been proposed for fabrication and optimization of FGPD devices. These findings offer insightful information to achieve high performance FGPD via process optimization.

\section{A brief introduction and properties of graphene}

Graphene is a 2D allotrope of carbon-made out of carbon atoms arranged on a honeycomb structure and can be thought of as benzene rings stripped out from their hydrogen atoms [17]. Although graphene is the mother for other allotropes of carbon such as fullerenes [18], carbon nanotubes [19, 20], and graphite [21] and has been presumably produced every time someone writes with a pencil, it took 440 years after its invention in order to isolate the graphene [22]. There were two main reasons for this delay, first, there was no expectation for existence of the free-state grapheme; second, there was lack of experimental tools existing to search for one-atom-thick flakes among the pencil debris covering macroscopic areas.

From the atomic point of view, graphene is made of $\mathrm{sp}^{2}$ hybridization carbon atoms between one $\mathrm{s}$ orbital and two $\mathrm{p}$ orbitals which form a trigonal planar structure with the formation of a $\sigma$ bond between carbon atoms that are separated by $1.42 \times 10^{-10} \mathrm{~m}$ [23]. Lattice structure of graphene is made out of two interpenetrating triangular lattices. The lattice unit vectors and nearest-neighbor vectors are indicated by $a_{1}$, and $a_{2}$, and $\delta, i=1,2,3$, respectively in Fig. 1(a).

The lattice vector of graphene with a trigonal planar structure with a basis of two atoms per unit cell can be written as [24]

$$
a_{1}=\frac{a}{2}(3, \sqrt{3}), a_{2}=\frac{a}{2}(3,-\sqrt{3})
$$

where $a$ is the carbon-carbon distance $\left(\approx 1.42 \times 10^{-10} \mathrm{~m}\right)$ and the reciprocal lattice vectors can be expressed as

$$
b_{1}=\frac{2 \pi}{3 a}(1, \sqrt{3}), b_{2}=\frac{2 \pi}{3 a}(1,-\sqrt{3}) .
$$

The points $k$ and $k^{\prime}$ on the corners of Brillouin zone [Fig. 1(b)] are of particular interest which are known as Dirac or neutrality points [25]. $k$ and $k^{\prime}$ positions in the momentum space can be expressed as

$$
k=\left(\frac{2 \pi}{3 a}, \frac{2 \pi}{3 \sqrt{3 a}}\right), k^{\prime}=\left(\frac{2 \pi}{3 a},-\frac{2 \pi}{3 \sqrt{3 a}}\right) .
$$

The three nearest neighbor vectors in the real space are given by

$$
\delta_{1}=\frac{a}{2}(1, \sqrt{3}), \delta_{2}=\frac{a}{2}(1,-\sqrt{3}), \delta_{3}=-a(1,0)
$$

and the six second-nearest neighbors are located at

$$
\delta_{1}^{\prime}= \pm a_{1}, \delta_{2}^{\prime}= \pm a_{2}, \delta_{3}^{\prime}= \pm\left(a_{2}-a_{1}\right) .
$$

The tight-binding Hamiltonian for electrons in graphene for nearest- and next-nearest-neighbor atoms implies that the energy bands derived from the Hamiltonian have the form

$$
a \pm \gamma_{0} \sqrt{1+4 \cos \frac{\sqrt{3} k_{x} a}{2} \cos \frac{k_{y} a}{2}+4 \cos ^{2} \frac{k_{y} a}{2}}
$$

where $a=\sqrt{3 a}$, and $\gamma_{0}$ is the transfer integral between first-neighbor $\pi$-orbitals (typical values for $\gamma_{0}$ are $\left.2.9 \mathrm{eV}-3.1 \mathrm{eV}\right)$. The $k=\left(k_{x}, k_{y}\right)$ vectors in the first Brillouin zone constitute the ensemble of available electronic momenta. The three $s, p_{x}$, and $p_{y}$ electrons fill the low-lying $\sigma$ band, the (-) band (negative energy branch) in (6) is fully occupied, whereas the $(+)$ branch is totally empty. Due to the Pauli principle, these filled shells form a deep valence band. These in-plane $\sigma$ bands are responsible for the robustness of the lattice structure in all allotropes.

The unaffected $p$ orbital can covalently bind with neighboring carbon atoms, leading to the formation of a $\pi$ band [Fig. 1(c)]. Since each $p$ orbital has one extra electron, the $\pi$ band is half filled [25]. These occupied and unoccupied bands 
touch at $k$ point on the corner of Brillouin zone. Therefore, as it is shown in Fig. 1(d), graphene is a zero-band-gap semiconductor. Expanding (6) at Dirac points yields the linear $\pi$ - and $\pi^{*}$-bands for Dirac fermions:

$$
E \pm(k)= \pm \hbar v_{f}|k|
$$

where $E$ is the energy bands, $h$ is plank's constant, $k=k-K$, and $v_{f}$ is the electronic group velocity, which is given by $\sqrt{3 \gamma_{0}} a /(2 \hbar) \approx 10^{6} \mathrm{~m} / \mathrm{s}$.



(a)

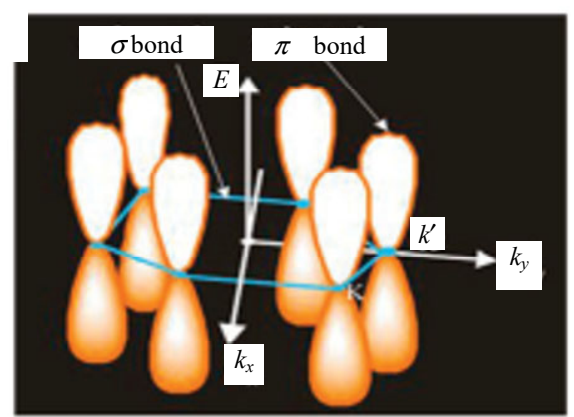

(c)

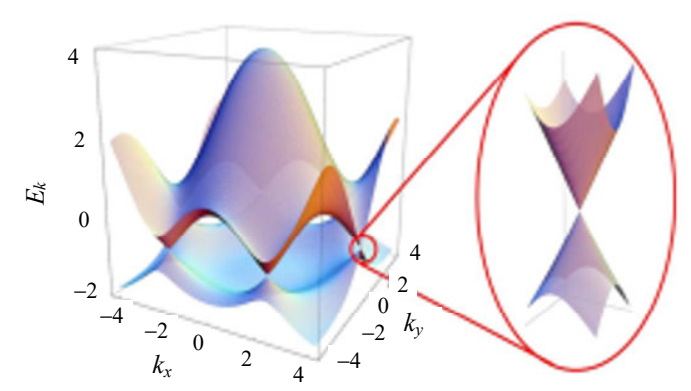

(d)

Fig. 1 Lattice vectors and bands in the graphene [25]: (a) graphene honeycomb lattice structure, (b) corresponding Brillouin zone. The Dirac cones are located at $k$ and $k^{\prime}$ points, (c) $\sigma$ and $\pi$ bonds in graphene, and (d) the band structure of graphene.

In graphene, the confining potentials are generated by various sources of disorders in the structure. The main sources of disorder are ionized impurities in the substrate, ad-atoms, and various molecules adsorbed in the graphene surface. Moreover, ripples in the graphene structure which is associated with the soft structure of graphene, lead to the phenomenon of Zitterbewegung, or jittery motion of the wave function. However, in graphene under certain conditions, Dirac fermions are immune to localization effects and therefore these electrons can propagate over large distances of the order of micrometers without suffering from scattering [26-28]. Graphene can be tailored chemically and/or structurally by techniques, such as deposition of metal atoms or molecules on its top surface, intercalation, incorporation of nitrogen, and/or boron in its structure as well as using different substrates that modify the electronic structure [29-32]. The properties of graphene which have been measured experimentally have not only exceeded those obtained in any other materials but also reached their theoretically predicted limits. A typical example is its room-temperature carrier mobility of $2.5 \times 10^{5} \mathrm{~cm}^{2} / \mathrm{V} \cdot \mathrm{s}$ which is found to be very close to the theoretical limit of $2 \times 10^{5} \mathrm{~cm}^{2} / \mathrm{Vs}$ [33] and $6 \times 10^{6} \mathrm{~cm}^{2} \cdot \mathrm{V}^{-1} \cdot \mathrm{s}^{-1}$ at $4 \mathrm{~K}[34]$. Other properties measured and reported in the literature are Young's modulus of $1 \mathrm{TPa}$ and intrinsic strength of $130 \mathrm{GPa}$ [35] which are very close to that predicted by theory [36], very high thermal conductivity of $2000 \mathrm{~W} \cdot \mathrm{m}^{-1} \cdot \mathrm{K}^{-1} \quad$ to $5300 \mathrm{~W} \cdot \mathrm{m}^{-1} \cdot \mathrm{K}^{-1} \quad$ [37], impermeability to any gases [38], optical absorption of exactly $p a<2.3 \%$ in the infrared limit [39], and extremely high densities of electric current (a million times higher than copper) [40]. It also exhibits specific integer and fractional quantum Hall effects [41, 42], a minimum electrical conductivity of $4 e^{2} / h$ even when the carrier concentration tends to zero, and Shubnikov-de Haas oscillations with a $\pi$ phase shift due to Berry's phase [43] which has characteristics of 2D Dirac fermions. From the electronics point of view, graphene crystals have two well-established allotropes, namely single layer graphene (SLG) and bilayer graphene (BLG), where 
in the former charge carriers resemble relativistic Dirac particles while in latter electrons also have some Dirac-like properties but a parabolic dispersion [44]. The transmittance of a free standing SLG can be derived by applying the Fresnel equations in the thin-film limit for a material with a fixed universal optical conductance $G_{0} \frac{e^{2}}{4 \hbar} \approx 6.08 \times 10^{-5} \Omega^{-1} \quad$ to give: $T=(1+0.5 \pi \alpha)$, where $\alpha=\frac{e^{2}}{4 \pi \varepsilon_{0} \hbar c} \approx \frac{1}{137}$ is the fine-structure constant [39]. Graphene only reflects $<0.1 \%$ of the incident light in the visible region, rising to $\sim 2 \%$ for ten layers [45].

(a)

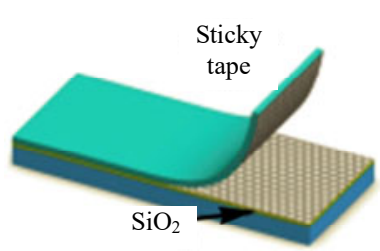

(d)

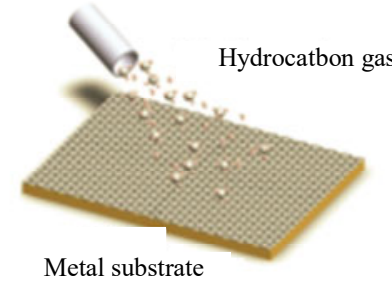

(b)

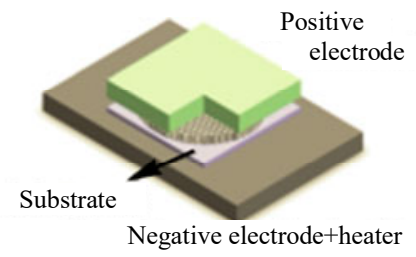

(e)

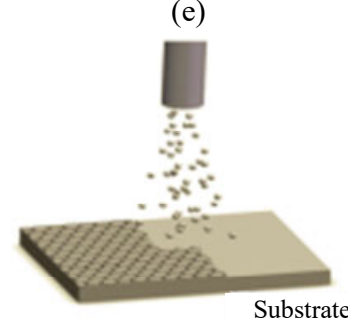

\section{Preparation techniques}

There are various preparation techniques for producing the graphene, ranging from the single layer to the multilayers. However, to choose an appropriate technique, it is important to have a perspective of design and application for the synthesized graphene. The current acceptable production techniques for the graphene growth include dry and wet exfoliation, chemical vapor deposition (CVD), physical vapor deposition (PVD), and growth on the silicon carbide $(\mathrm{SiC})$ substrate which will be discussed in this review. Here we don't go through the production of graphene oxide.

Fig. 2 Various graphene production techniques [52]: (a) mechanical exfoliation, (b) anodic bonding, (c) liquid phase exfoliation, (d) CVD process, (e) PVD process, and (f) growth from $\mathrm{SiC}$.

\subsection{Dry exfoliation}

Dry exfoliation is the splitting of layered material (LM) into atomically thin sheets via mechanical, electrostatic, or electromagnetic forces in air, vacuum or inert environments. Dry exfoliation can be categorized as mechanical exfoliation and anodic bonding.

Mechanical exfoliation includes peeling highly ordered pyrolytic graphite (HOPG) using the adhesive tape [Fig.2(a)]. For research purposes and new concept devices, mechanical exfoliation has been used by researchers for decades and particularly in the last two decades, which is implemented as a method of cleaving graphite, yielding films of several layers of graphene [46, 47]. According to the Vander Waals bonding of each layer of graphene to the other layer, it is feasible to cleave HOPG. Normally, the peeling is performed several times.

The anodic bonding method is inspired from a technique used for bonding Si to a Pyrex substrate $[48,49]$. In this approach, a potential difference of the order of $\mathrm{kV}$ is applied to a heated Pyrex/Si and consequently a permanent and irreversible bonding between the substrate and silicon is obtained. 


\subsection{Wet exfoliation}

Graphite can also be exfoliated using liquid-phase exfoliation (LPE) which uses a solvent to exfoliate graphite by ultrasonication to extract individual layers [Fig.2(c)]. Solvents such as acetic acid, sulfuric acid, and hydrogen peroxide are ideal for graphene dispersion since they minimize the interfacial tension between graphene flakes and liquid. Interfacial and surface tension plays a key role when a solid surface is immersed in a liquid medium. The latter can be defined as the property of the surface of a liquid that allows it to resist an external force, due to the cohesive nature of its molecules [50]. In the case of graphene and graphitic flakes, solvents with surface tension $\left(\gamma \sim 40 \mathrm{mN} \cdot \mathrm{m}^{-1}\right)$ are reported as the best option for the dispersion, since they minimize the interfacial tension between the solvent and graphene [51, 52]. $\mathrm{N}$-methylpyrrolidone (NMP) and dimethylformamide (DMF) as the solvents with $40 \mathrm{mN} \cdot \mathrm{m}^{-1}$ have some reported disadvantages such as toxicity and high boiling point low boiling point solvents, such as acetone and isopropanol, which have been proposed as an alternative because of low boiling point solvent which makes the process of solvent removal much easier [53]. Due to the very high surface tension $\left(72 \mathrm{mN} \cdot \mathrm{m}^{-1}\right)$ of about $30 \mathrm{mN} \cdot \mathrm{m}^{-1}$ higher than that of NMP, water is not a suitable solvent for dispersion of graphene and graphite. Exfoliation step can be done via chemical wet dispersion followed by ultrasonication, both in aqueous [54, 55] and non-aqueous solvents [56-58]. And the final step is the purification through ultracentrifugation to separate exfoliated from un-exfoliated flakes. Ultrasound-assisted exfoliation is a very common technique for producing cheap and easily scalable graphene flake and does not require expensive growth substrates [59]. After exfoliating, the inter-sheet attractive forces would balance through the solvent-graphene interaction. Graphene is best prepared as flakes, in order to maximize the active surface. The flakes can be used in various applications such as conducting inks [Fig. 3(a)] [60, 61], thin films [51, 62] [Fig. 3(b)], and composite materials [63] [Fig.3(c)]. In order to deposit these flakes, various techniques can be implemented which have been summarized in Figs. 3(d)-3(g). Drop and dip casting [Fig. 3(d)], rod [Fig. 3(e)], spray coating [Fig. 3(f)], screen and ink-jet printing [Fig. $3(\mathrm{~g})]$, vacuum filtration, and Langmuir-Blodgettare are the mostly used techniques. The main drawback of this technique is the limited size of produced flakes by this technique. To date, LPE-SLGs have area mostly below $1 \mu \mathrm{m}^{2}$.

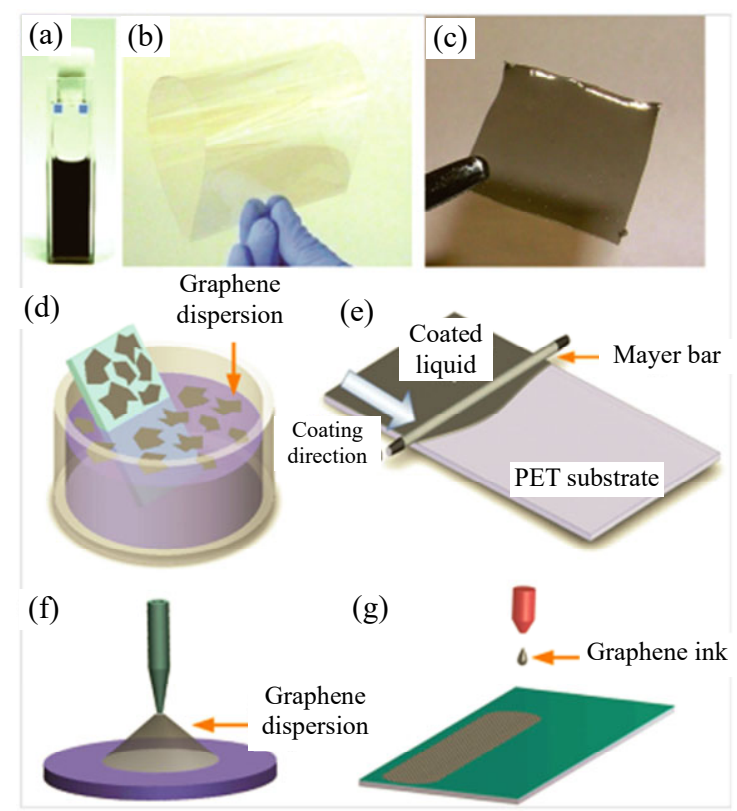

Fig. 3 Various applications of graphene flakes [52]: (a) conducting inks, (b) thin films, and (c) composite materials. Different techniques for deposition of flakes consist of (d) drop and dip casting, (e) rod, (f) spray coating, and (g) screen and ink-jet printing.

\subsection{Chemical vapor deposition (CVD)}

Currently, CVD is the most widely used deposition technique to obtain graphene [Fig.2(d)]. CVD processes can be categorized as thermal, plasma enhanced (PECVD), cold wall, hot wall, reactive, and many more. Thermal CVD of graphene involves the use of the first row of transition metals, such as iron (Fe) [64, 65], cobalt (Co) [66, 67], nickel $(\mathrm{Ni})[68,69]$, and copper $(\mathrm{Cu})[70-72]$ as catalyst in order to produce the large scale 
production of graphene. Carbon solubility among these metals is the main parameter which impacts the growth quality, where $\mathrm{Fe}$ has the highest and $\mathrm{Cu}$ has the lowest carbon solubility. Therefore, copper is the preferred catalytic metal to grow monolayer graphene, while nickel and cobalt usually end up by giving the monolayer to multilayers (up to 10 layers) of graphene [73] and Fe commonly used for the growth of few layers graphene (FLG). Graphene growth using CVD is a fairly straightforward technique where a fixed ratio gas mixture of hydrogen/methane $\left(\mathrm{H}_{2} / \mathrm{CH}_{4}\right)$ is introduced in the CVD chamber and heated up to $1000{ }^{\circ} \mathrm{C}$. Carbon is extracted from the methane gas through thermal dissociation and catalyzed on the copper surface where graphene is formed. Prior to the introduction of carbohydrate gas (methane), a high temperature annealing at approximate temperature of $1000{ }^{\circ} \mathrm{C}$ in hydrogen reduces atmosphere in necessary to enables the growth of grain boundaries [74, 75]. It is also expected that crystal domains within the copper foil will soften up and merge with each other to form a larger domain size since the melting point of copper is about $1085{ }^{\circ} \mathrm{C}$. Moreover, it eliminates any oxide layer $\left(\mathrm{CuO}\right.$ and $\left.\mathrm{Cu}_{2} \mathrm{O}\right)$ present in the metal which may reduce the catalytic activity of the copper foils. Plasma enhancement CVD (PECVD) is a subcategory of the CVD technique for the synthesis of graphene. This approach is based on a number of plasma sources, such as microwave (MW) [76], radio frequency (RF) [77], and direct current (DC) arc discharge [78]. It should be noted that, the electrical properties of the graphene obtained through the CVD method are limited due to the graphene transfer process during the device fabrication. It has been shown in $[79,80]$ that after the transfer process, the electrical properties of graphene are degraded compared with the mechanically exfoliated graphene (pristine graphene). However, CVD technique still has an advantage in terms of being able to deliver large area and high-quality grapheme (coverage areas greater than those of $95 \%$ have been reported) [74].

\subsection{Physical vapor deposition}

The processes of depositing materials directly from the vapor phase known as physical vapor deposition (PVD) comprise the following methods: evaporation, sputtering, and ion plating as well as the reactive types of these processes [Fig. 2(e)]. There have been a few reports on the use of cathode arc deposition and radio frequency sputtering [81-84], pulsed laser deposition (PLD) [85], and pulsed DC magnetron sputtering (PMS) [86] for the synthesis of SLG and FLG. Hydrogen arc discharge is used as a rapid heating method to produce graphene sheets (GSs) with a good quality from graphene oxide (GO). This method mainly involves three key steps: oxidation of graphite, exfoliation of the GO by hydrogen arc discharge, and finally the dispersion of exfoliated graphite (EG) to obtain GSs by ultrasonication [87]. In another approach, Deng et al. [81] implemented radio frequency (RF) hydrogen plasma sputtering without the help of a catalyst and carbonaceous gas to deposit FLG on various substrates. They have discussed the growth of FLG based on a defect nucleation and diffusion growth mechanism. PLD is another simple physical deposition process in which a laser is used as a source of pure form of energy to ablate materials from the surface of a target. Pulsed lasers have been employed for growth of carbon nanostructures of different dimensions, including fullerenes [88], carbon nanotubes [89], graphite, and diamond-like carbon [90, 91]. The fabrication of controlled number of graphene layer using PLD technique was reported by Zhang et al. [92] using excimer laser on transition metal templates as catalyst. Recently, the growth of FLG by PLD on nickel (Ni) substrate under the stationary magnetic field effect at room temperature has also been reported [93]. Recently, multilayer and single layer graphene have been grown on copper foil using a bipolar pulsed direct current (DC) magnetron sputtering (PMS) of a graphite target in pure Ar atmosphere [94]. This technique has developed by combining the benefits of both DC and RF magnetron sputtering. 


\subsection{Epitaxial growth on silicon carbide substrate}

Acheson reported a method of producing graphite from $\mathrm{SiC}$ in 1896 for lubricant applications [95]. In order to reduce the defects like misfit dislocations during graphene growth, it is desirable to have a lattice matched iso-structural substrate. However, for both $4 \mathrm{H}-\mathrm{SiC}$ (Si-face) and $6 \mathrm{H}-\mathrm{SiC}$ (C-face) the lattice mismatch between graphene and $\mathrm{SiC}$ is also very large $(\sim 25 \%)$. Due to the weak bonding between the grown graphene and the $\mathrm{SiC}$ substrates, $\mathrm{SiC}$ is described as a highly non-lattice-matched substrate. Therefore, this layer can acts as a buffer layer. In this case, because of the anisotropic nature of their chemical bonds, the films grow parallel to the substrate. The growth rate of graphene on $\mathrm{SiC}$ depends on the specific polar $\mathrm{SiC}$ crystal face [Fig.2(f)]. A disadvantage of graphene growth on the $\mathrm{SiC}$ substrate for large-scale production is the cost of $\mathrm{SiC}$ wafers and their smaller size compared with Si wafers. Finally, it should be noted that despite the multitude of available growth techniques mentioned above, still a single reliable method for obtaining a large area, high quality graphene sheets, with the minimum grain boundaries grown directly on an insulator, has not been introduced.

\section{Graphene transfer techniques}

In most of the cases, for obtaining the best performance from the graphene devices, it is crucial to transfer graphene films from the grown substrate to the target substrate with the large-area, clean, and low-defect surface. Tearing and ripping of the graphene sheets during the transfer process are the main reasons for degradation in the quality of the grown graphene [96, 97]. Here, the most common transfer methods of graphene which are based on various polymers and etchants are discussed, then the innovative techniques which have been introduced to overcome the problem associated with etchant and polymer based transfer of graphene is explained, and the latest transfer techniques with less impact on the quality of transferred graphene are presented. Finally, a few works on transferring free synthesis are mentioned. It should be noted that the transfer of HOPG using mechanical exfoliation $[22,98-100]$ is not discussed here.

As it is alrady mentioned, among various synthesis techniques, CVD growth of graphene on metal is believed to be an efficient method for large area graphene growth with a good quality. A number of metals such as Fe [64, 101], Ru [102, 103], Co [104], Ir [105, 106], Pt [107], Au [108], Ni [109], and $\mathrm{Cu}[110]$ are used for catalytic purposes during the growth process. These metal substrates are typically unwanted after the synthesis is completed so the removal of the metal is the logical step after the growth. Among these metals, $\mathrm{Ni}$ and $\mathrm{Cu}$ are preferred since they are the most economical metal substrates and also the etching process of them is straightforward. Therefore, we mainly focus on transfer of graphene grown on $\mathrm{Ni}$ and $\mathrm{Cu}$ substrates.

Polydimethylsiloxane (PDMS) is amongst the first polymers which are used for CVD graphene transfer. Its unique properties such as durablility, unreactivity, moldablility, and resistance to many solvents make it an excellent candidate for graphene transfer, however the most significant quality of PDMS is the low surface free energy. In order to transfer graphene by PDMS, it is brought into contact with graphene on a metal substrate. After the metal etching step, the released graphene on PDMS can be moved to a target substrate which is typically polyethylene terephthalate (PET) or $\mathrm{SiO}_{2} / \mathrm{Si}$. At this step, the graphene is released from PDMS and transferred to the target due to the low adhesion force that PDMS maintains with the substance. This releases the graphene from PDMS and stamps it onto the substrate. In Fig. 4, the schematic process of the graphene transfer via PDMS is shown. One of the drawbacks of using PDMS based transfer of graphene, particularly for large area graphene synthesis, is the long etching time. 
(a)

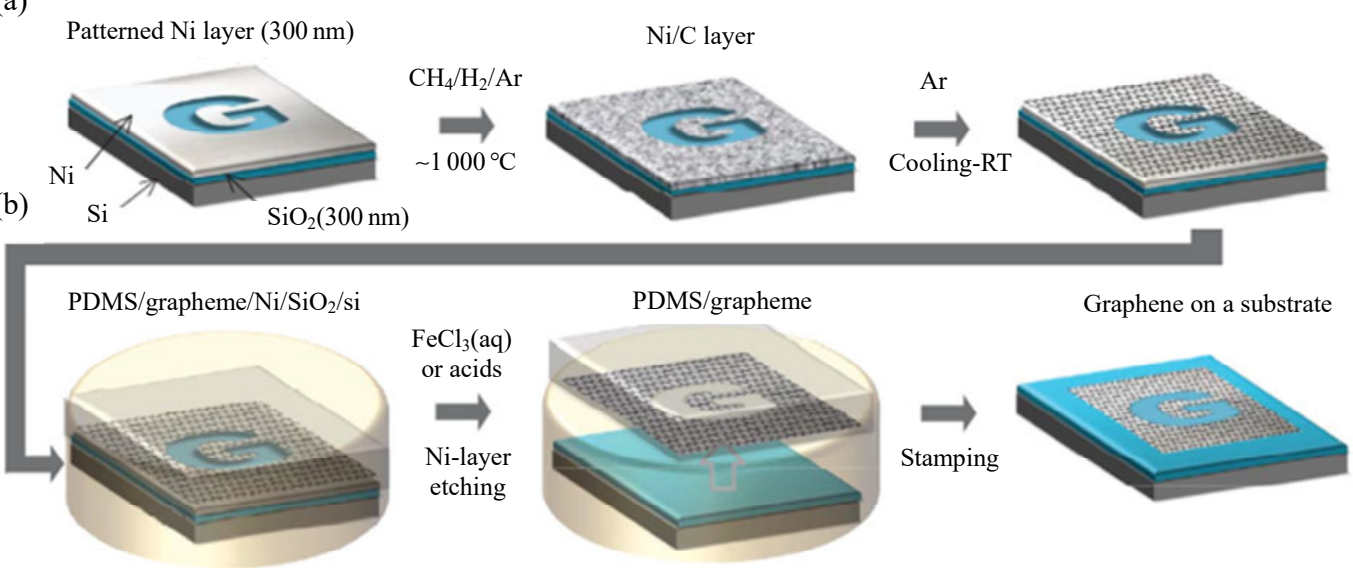

Fig. 4 Deposition, etching, and transfer processes of grapheme [109]: (a) growth of graphene on thin nickel layer and (b) etching the Ni layer using $\mathrm{FeCl}_{3}$ (or acids) and transferring it to a substrate by using a PDMS stamp.

Polymethyl methacrylate (PMMA) is another widely used polymer support for graphene transfer due to its prominent features, such as flexibility, good solubility in several organic solvents, decomposability above $250{ }^{\circ} \mathrm{C}$, the relatively low viscosity $\left(43.4 \mathrm{mPas}\right.$ at $25{ }^{\circ} \mathrm{C}$ ), excellent wetting capability, and most importantly the high transparency of the PMMA which makes it clear to observe the process of metal removal [111]. The usual process for graphene transfer by PMMA is depositing a PMMA sacrificial layer on the graphene/metal surface and subsequently etching the underlying metal by an appropriate etchant solution. Typical etchant of $\mathrm{Ni}$ and $\mathrm{Cu}$ metals are iron nitrate $\left[\mathrm{Fe}\left(\mathrm{NO}_{3}\right)_{3}\right]$, iron chloride $\left(\mathrm{FeCl}_{3}\right)$, and ammonium persulfate $\left[\left(\mathrm{NH}_{4}\right)_{2} \mathrm{~S}_{2} \mathrm{O}_{8}\right]$. Strong acids such as $\mathrm{HNO}_{3}$ often produce hydrogen bubbles which damage the graphene. Aqueous iron (III) chloride $\left(\mathrm{FeCl}_{3}\right)$ solution as an oxidizing etchant is the most commonly used etchant to remove the nickel layers. The ionic equation of the etching reaction can be represented as follows:

$$
2 \mathrm{Fe}^{3+}(\mathrm{aq})+\mathrm{Ni}(\mathrm{s}) \rightarrow 2 \mathrm{Fe}^{2+}(\mathrm{aq})+\mathrm{Ni}^{2+}(\mathrm{aq}) .
$$

According to this redox process, the nickel layers etches effectively within a mild $\mathrm{pH}$ range without forming gaseous products or precipitates. Then, the graphene film separates from the substrate and floats on the surface of the solution after a few minutes of process [Figs. 5(a) and 5(b)]. This film is then ready to be transferred to any kinds of target substrates [Figs. 5(c) and 5(d)] [109].

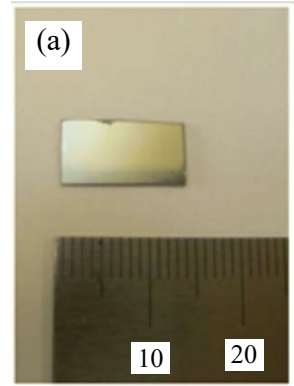

(c)

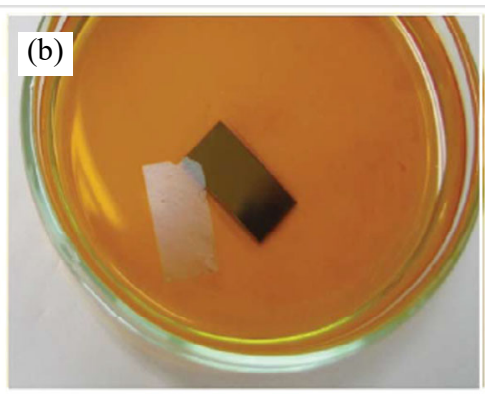

(d)

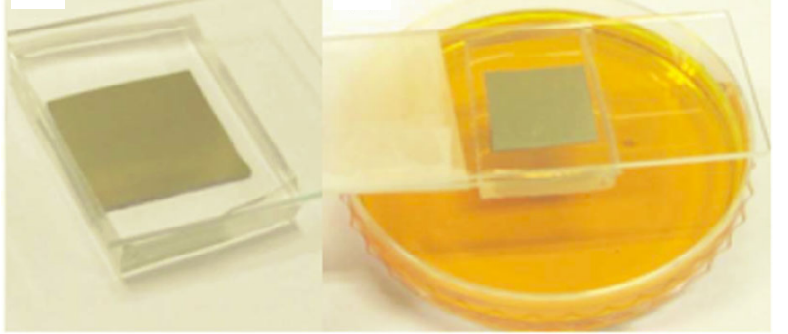

Fig. 5 Transfer process for large-scale graphene films [109]: (a) graphene film grown on a $\mathrm{Ni}(300 \mathrm{~nm}) / \mathrm{SiO}_{2}(300 \mathrm{~nm}) / \mathrm{Si}$ substrate; (b) a floating graphene film after etching the nickel layers in $\mathrm{FeCl}_{3}$ aqueous solution; (c) and (d) the transfer based on a PDMS stamp for transferring the patterned graphene films.

The PMMA/graphene needs to be washed by de-ionized (DI) water to remove the trace of etchant. The combination of PMMA and graphene then transfers to the target substrate and finally the PMMA layer is removed by acetone, and then washed by DI water and dried [68]. Li et al. [97] modified this technique of the graphene transfer by 
PMMA through adding a second PMMA coating step after the PMMA/graphene was placed on the target substrate $\left(\mathrm{SiO}_{2} / \mathrm{Si}\right)$.

Figure 6 shows the original and modified technique of graphene transfer using PMMA. The role of the second layer of PMMA is to allow the pre-coated PMMA to partially or fully dissolve and mechanically relax the underlying graphene. Poly (bisphenol A carbonate) (PC) [112], polyisobutylene (PIB) [113], poly(lactic acid) (PLA), and poly(phthalaldehyde) (PPA) [114] are other polymers which have been used as the supporting layer in the graphene transfer. Recently, Matsumae et al. [115] introduced a clean graphene transfer process by employing polymethylglutarimide (PMGI)-based resist as a support scaffold. This method is mainly based on using a lift-off resist (LOR) technique introduced by MicroChem [116] after photolithographic processing for obtaining clean graphene. Various techniques are implemented to obtain clean graphene surface after the polymer removal. For rigid substrates, annealing in high temperature $\left(250^{\circ} \mathrm{C}-350^{\circ} \mathrm{C}\right)$ [117] can be an efficient way to remove the polymer residues. Park et al. [118] used approaches such as immersing the graphene/PMMA in acetone vapor to minimize tearing of graphene by direct immersion in acetone solution, and brief acetone dipping for $2 \mathrm{~min}$, followed by $3 \mathrm{~h}$ of annealing at $500^{\circ} \mathrm{C}$ under the protecting gas mixtures of hydrogen and argon to remove PMMA on graphene. Using an organic buffer layer between the PMMA and graphene [119], ultra violet (UV) irradiation for degradation of PMMA, and using mixed solvent of isopropyl alcohol (IPA), acetone, and methyl isobutyl ketone (MIBK) [120] have also been reported for PMMA residue removal. Recently, transfer methods known as polymer-free graphene transfer have also been introduced to ignore the drawbacks of polymer based transfer such as thermal stress that causes damage to the graphene without completely removing the polymer and consequently varies the electronic properties and band structures of graphene. Ragan et al. [121] directly transferred graphene from $\mathrm{Cu}$ growth substrates to holey amorphous carbon (a-C) transmission electron microscopy (TEM) grids through two processes of surface tension and evaporation.



Fig. 6 Modified approach for transfer of graphene films. The optical micrographs of graphene transferred on $\mathrm{SiO}_{2} / \mathrm{Si}$ wafers with "bad" and "good" transfers are shown on top-right and bottom-left, respectively. A $4.5 \times 4.5 \mathrm{~cm}^{2}$ graphene on the quartz substrate also presented in the bottom-right image [97].

The roll-to-roll transfer technique is another successful transfer technique for large area graphene grown on copper and is based on the use of thermal release tape as the supporting layer [75]. The process starts with heat treatment of copper foils to increase the grain size. After the growth of graphene using CVD, the graphene film grown on copper foil attached to a thermal release tape by applying an appropriate pressure between two rollers. After etching the copper substrate, a roller at the modest temperature of $120{ }^{\circ} \mathrm{C}$ is used to attach the graphene film on the thermal release tape and target substrate. The heat removes the adhesiveness of the tape and the released graphene adheres to the target substrate [Fig. 7(a)]. Using this approach, group successfully reported transfer of graphene with 30-inch diagonal dimension [Fig. 7(b)]. 


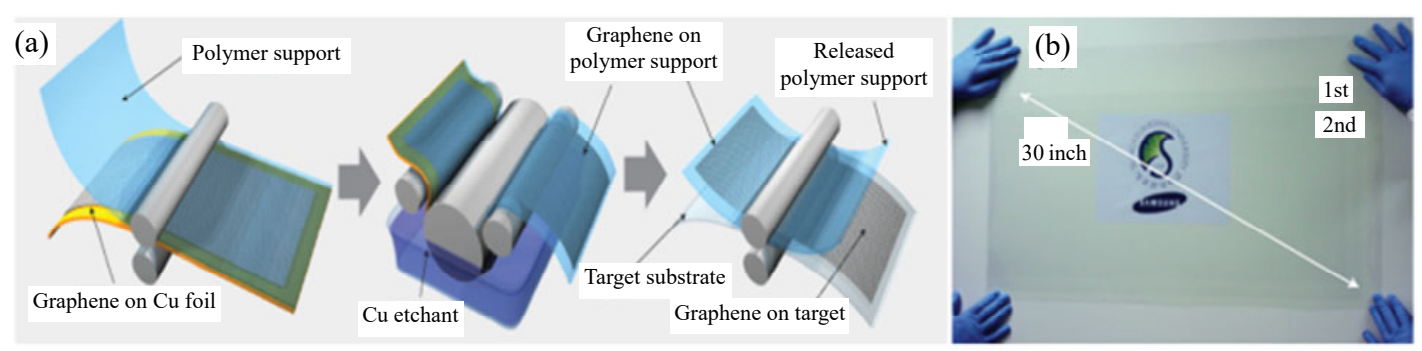

Fig. 7 Production of large-scale, continuous graphene film on flexible substrate through roll-to-roll technique [75]: (a) the process of roll-based transfer includes attachment of a polymer support on graphene/Cu foil, copper etching, and dry-transfer-printing on a target substrate and (b) a transparent 30-inch graphene film transferred on a 35-inch PET sheet.

In addition to the problems mentioned for polymer residue on the graphene surface, there are a few problems such as unintentional chemical doping introduced in the graphene structure during metal substrate etching [122]. Therefore, suppression of etchant effect on the graphene structure or even eliminating this step seems favorable.

Electrochemical delamination process is also an interesting approach used for the graphene transfer. In this technique, same as normal transfer technique, a PMMA layer acts as a supporting layer for grapheme grown on the metal. In the case of CVD grown graphene on $\mathrm{Cu}$, a solution of Potassium persulfate $\left(\mathrm{K}_{2} \mathrm{~S}_{2} \mathrm{O}_{8}\right)$ in an electrolytic cell is used as the electrolyte and PMMA/graphene/Cu and glassy carbon are used as the cathode and anode, respectively [Fig. 8(a)]. The hydrogen gas bubbles emerging between the graphene and $\mathrm{Cu}$ layer along with slight etching of $\mathrm{Cu}$ induces lift-off of the PMMA/graphene [Figs. 8(b) to 8(d)] [71].

(a)

(c)
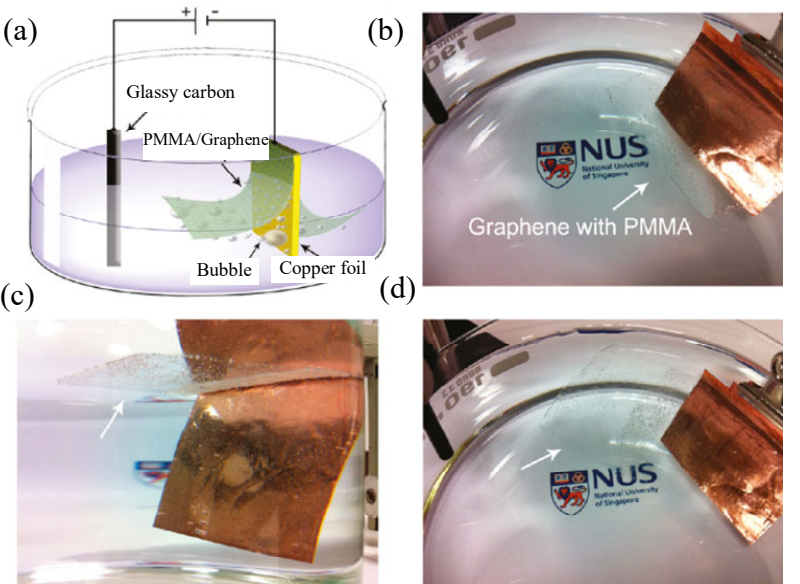

(d)



Fig. 8 Electrochemical exfoliation of graphene from copper foil [71]: (a) schematic presentation of electrochemical cell and (b)-(d) optical images showing the details of peeling PMMA/graphene from the $\mathrm{Cu}$ foil.
This method particularly can be used for graphene grown on noble metals such as Ir, Pt, and $\mathrm{Au}$, in which it is expensive and difficult to etch. Although the above techniques improve the quality of transferred graphene by suppressing the impact of polymer residue and metal etchants, the need for the growth techniques without requiring the graphene transfer (transfer free growth) is highly desirable. Several groups have investigated techniques to directly grow graphene on the target substrate [84, 123-131]. Most of the transferred free graphene growth approaches are based on the existence of catalyst metallic layers such as $\mathrm{Ni}, \mathrm{Cu}$, or $\mathrm{Co}$. In one of the first attempts, Levendorf et al. [123] used a copper to grow SLG for device application. Their basic mechanism was not novel; however, they used an evaporated copper film instead of a copper foil and a thin $\mathrm{Ni}$ adhesion layer, which were both directly evaporated onto a $\mathrm{SiO}_{2} /$ silicon wafer.

In this section, various transfer techniques of graphene are reviewed and the pros and cons of each method are mentioned. It is obvious that still there is no clear winner for the best transfer technique. The problems of the transfer technique are more obvious when we deal with the large area graphene transfer. Therefore, for future application of graphene, improving synthesis techniques and transfer methods is crucially important. Although, there are reports on transferring free graphene growth on the target substrate, still the conventional transfer mechanisms are the prior option in the applications of graphene before the complete maturity of transfer free techniques. 


\section{Photodetectors}

Photodetectors are devices that measure photon flux or optical power by converting optical signals into electrical current through electronic processes. Principally, the mechanism of photo detection includes three processes: (1) generation of carriers by incident light, (2) carrier transport or multiplication through the current-gain mechanism, and (3) extraction of carriers as terminal current to provide the output signal [132]. We can categorize the photodetectors by various structural and operational parameters. For instance, based on the mechanisms of the photo-generated carrier's collection, photodetectors can be divided into photoconductor and photovoltaic detectors (photodiodes). In photoconductors under illumination, the photo-generated carriers change the conductivity of the device and an external bias is required to separate and sweep these photogenerated carriers to the device contacts. The transport mechanism of a photoconductor is dominant by the majority drift carriers. On the other hand, in the photovoltaic mode, the detector usually operates at reverse or zero bias condition and the photo-generated carriers are separated by the built-in electrical field of a p-n junction, and the majority carriers on both sides are blocked by the built-in potential barrier. Therefore, the transport mechanism of these devices is dominated by the diffusion of the minority carriers. The most available photodetectors in the market are semiconductors photodetectors which work based on the quantum photoelectric effect; a photon excites a carrier which contributes to the photocurrent. There is another class of photodetectors of which the working principal is more similar to the thermal sensors and is suitable for far-infrared wavelengths. The efficiency of the photodetectors is determined by external and internal quantum efficiencies, noise equivalent power (NEP), specific detectivities $\left(D^{*}\right)$, life time, etc. Quantum efficiency is the number of electron-hole $(\mathrm{E}-\mathrm{H})$ pairs generated per incident photon. The external and internal quantum efficiency (E/I QE) are defined as the number of $(\mathrm{e}-\mathrm{h})$ pairs per second collected to produce the photocurrent $I_{\mathrm{ph}}$, divided by the number of incident/absorbed photons per second and can be expressed as $E_{\mathrm{QE}}=\left(I_{\mathrm{ph}} / q\right) / \varphi_{\mathrm{in}}$ and $I_{\mathrm{QE}}=\left(I_{\mathrm{ph}} / q\right) / \varphi_{\text {abs. }}$. In these equations, $q$ is the electron charge, $\varphi_{\text {in }}=$ $P_{\mathrm{in}} / E_{\mathrm{ph}}$, and $\varphi_{\mathrm{abs}}=\varphi_{\text {in }} A_{\mathrm{abs}}$, where incoming photon flux $\varphi_{\text {in }}$ and absorbed photon flux $\varphi_{\text {abs }}$ are related to incident photon energy $E_{\mathrm{ph}}$ and power $P_{\text {in }}$ and absorbed fraction. The responsivity of a photodetector is the ratio of photocurrent $I_{\mathrm{ph}}$ and incident power: $R_{\mathrm{ph}}=I_{\mathrm{ph}} / P_{\text {in. }}$. The $N E P$ is a function of noise and responsivity. NEP is defined as the value of the root mean square (RMS) input radiant signal power required to produce an rms output signal equal to a RMS noise value with a signal to noise ratio (SNR) of 1 and usually expressed in units $W$ per $\sqrt{\mathrm{Hz}}$ [133]. $D^{*}$ is another parameter of interest which is reversely proportional to $N P E$. Detectivity, defined as $D^{*}=\sqrt{(A \cdot B W)} / N E P$, is measured in $\mathrm{cm} \sqrt{\mathrm{Hz}} / W$ where $A$ is the area of the photosensitive region, and $B W$ is the frequency bandwidth of the detector. The incoming photon flux $\varphi_{\text {in }}$ and carrier density $\Delta n$ change, are linearly proportional by equation $\Delta n=\tau_{t r} \times Q E \times \varphi_{\text {in }}$. In this equation, $\tau_{\mathrm{tr}}$ is the lifetime of the charge residing in the particles and $Q E$ is the external quantum efficiency which is defined as $Q E=\eta_{\text {trans }} \eta_{\text {abs, }}$, where $\eta_{\text {trans }}$ is the charge transfer efficiency and $\eta_{\text {abs }}$ is the light absorption efficiency. Based on the mentioned figure of merit, basic requirements for a modern photodetection platform are high performance in terms of speed, efficiency or wavelength range, low noise for reduction error, sufficient area for coupling to optical fiber, flexibility, transparency, and complementary metal-oxide-semiconductor (CMOS) integrability.

Although conventional semiconductors are of great advantages for fabrication of photodetectors, there are some limitations for implementation in the modern devices. In [134], various photodetectors 
based on nanostructures in wide-range of electromagnetic spectrum were classified based on major parameters of operation. One of the most challenging issues associated with $\mathrm{Si}$ (dominated material in solid-state electronics and photonic devices in last four decades) is the intrinsic band gap which restricts the photoresponsivity of device to specific light bandwidth. Silicon and cadmium sulfide $(\mathrm{CdS})$ nano-ribbon photodetectors can be used mainly for detection in the visible regime of electromagnetic spectrum; gallium sulfide (GaS) nanosheet and molybdenum disulfide $\left(\mathrm{MoS}_{2}\right)$ enable the detection in ultraviolet and infrared regimes, respectively. Small bandgap semiconductor compounds such as mercury cadmium telluride (HgCdTe), lead sulfide $(\mathrm{PbS})$ or lead selenide $(\mathrm{PbSe})$ were used for detection of mid-infrared photons, and in the far-infrared regime the thermal sensing techniques are utilized. In addition to the limitations associated with the bandwidth, the speed of response in conventional photodetectors is limited by capacitative effects, the trapping of charge carriers, and the saturation speed of carriers which suppress the response of the photodetector in the high-frequency domain. In another classification and from the structural point of view, the most conventional photodetectors can be classified as $p-n$ junction photodiodes, $\mathrm{p}-\mathrm{i}-\mathrm{n}$ photodiode (a special case of $\mathrm{p}-\mathrm{n}$ junction), and Schottky junction photodetectors [Schottky barrier photodiodes and metal semiconductor-metal (MSM) photodiodes]. In Fig. 9, device configurations of some high-speed photodiodes as the most-common photodetectors are presented. Principally, in photodiodes the formation of depleted region in the semiconductor and the corresponding high electric field region in that area serves to separate photogenerated E-H pairs. Always there is a trade-off between the speed of response and quantum efficiency since on one hand to reduce the transit time the depletion region must be kept as thin as possible, while in order to increase the quantum efficiency, this region of depletion must be sufficiently thick to allow a large fraction of the incident light to be absorbed.

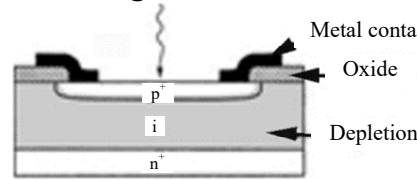

(a)

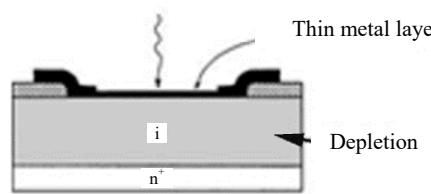

(c)

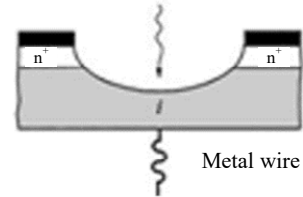

(e)



(b)

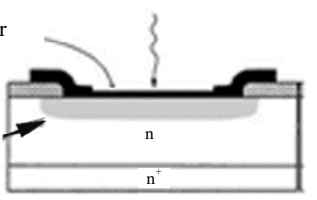

(d)
Fig. 9 Device configuration of some high-speed photodiodes as the most-common photodetectors [132]: (a) p-i-n photodiode, (b) p-n photodiode, (c) metal-i-n photodiode, (d) metal-semiconductor photodiode, and (e) point-contact photodiode.

Alternative materials, such as $2 \mathrm{D} \mathrm{MX}_{2}$ type crystals [135], transition-metal dichalcogenides (TMDs) [136, 137], transition oxides [138, 139], silicone [140], germanene [141, 142], perovskite materials (e.g., $\left.\left[\mathrm{NH}_{3}\left(\mathrm{CH}_{2}\right)_{12} \mathrm{NH}_{3}\right] \mathrm{PbX}_{4}\right)$ [143, 144], are also utilized as the active part of photodetectors.

\subsection{Graphene photodetectors and photo-detection mechanisms}

Graphene has the capability to play the role of main building material in novel photonic and optoelectronic devices and particularly advanced photodetectors due to its fascinating properties. Among the introduced devices for photodetection purpose, graphene based photodetectors are of great importance since single layer graphene (SLG) is a gapless material which enables photogeneration of carriers in the wide range of electromagnetic spectral regime, including ultraviolet, visible, short-wave infrared (SWIR), near-infrared (NIR), mid-infrared (MIR), far-infrared (FIR), and terahertz $(\mathrm{THz})$. Furthermore, graphene exhibits ultrafast carrier dynamics $[145,146]$, tunable optical properties via electrostatic doping [147, 148], low 
dissipation rates, high mobility, and the ability to confine electromagnetic energy to unprecedented small volumes. The high carrier mobility enables ultrafast conversion of photons or plasmons to electrical currents or voltages [149]. Over the past few years, various graphene based photodetectors structures, such as metal-graphene-metal (MGM) photodetectors, graphene p-n junction photodetectors, grapheme-semiconductor heterojunction photodetector, hybrid photodetectors, and integration of graphene into an optical micro-cavity or wave guides have been investigated in the literature. Before we go through the details of each class of photodetectors, we briefly explain the various photo-detection mechanisms which are the basis of operation in these devices. In the case of graphene, mechanisms, such as photovoltaic [Fig. 10(a)], photo-thermoelectric [Fig. 10(b)], bolometric [Fig. 10(c)], photo-gating effect, and Dyakonov-Shur (DS) or plasma-wave-assisted mechanism [Fig. 10(d)], are the dominant mechanisms in the photocurrent generation and consequently photodetection processes. Photovoltaic (PV) photocurrent generation is based on E-H pair formation and acceleration in opposite directions and built-in electric fields at junctions between negatively (n-type) and positively (p-type) doped regions of graphene. If the hot photogenerated carriers reach to the contacts [source (S), drain (D)], or if it establishes a local photovoltage within the focal area, then the photocurrent would be generated.

There are a few methods for formation of built-in-electric field between the regions such as the doping by local chemical doping [150], use of two (split) gates $(G)$ which electrostatically modify the doping under the gate region $[151,152]$, or by formation of a work-function difference between graphene and a metal contact $[153,154]$. The direction of built-in-electric field determines the direction of photocurrent and the doping level is not an important parameter. If a temperature gradient is generated by laser light across the junction of two materials with different thermoelectric powers (S), there is photocurrent generation by the photo-

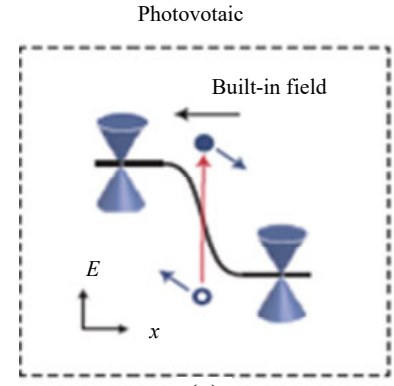

(a)

Bolometric

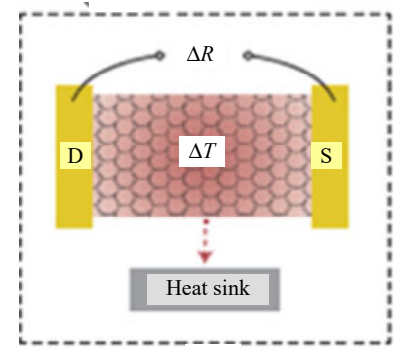

(c)

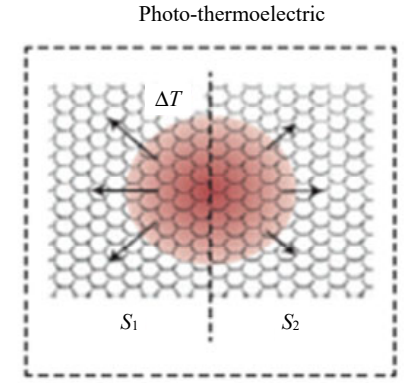

(b)

Dyakonov-Shur

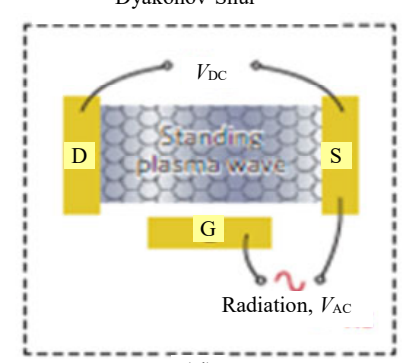

(d)
Fig. 10 Various photocurrent generation mechanisms [149]: (a) photovoltaic, (b) photothermoelectric, (c) bolometric, and (d) plasma wave-assisted (Dyakonov-Shur).

thermo-electric effect (PTE). In graphene, hotcarrier-assisted transport is a dominant mechanism of carrier transport. Hot carriers created by the radiation field can remain at a temperature higher than that of the lattice for many picoseconds (ps) because of the large optical phonon energy $(\approx 200 \mathrm{meV})$ in graphene [155] and they go to the equilibrium with the lattice via the slower scattering between carriers and acoustic phonons. In a system with various graphene layer numbers due to the various dispersion relations, the densities of states (DOS) are different. For instance, in a system of a single and bilayer graphene [Fig. 11(a)] the DOS of single-layer is smaller than that of the bilayer. Accordingly, a built-in-potential difference forms between two regions due to the larger fermi energy of a single-layer than the bilayer. On the other hand, the Dirac point of the single layer is lower than the bilayer because of fermi level alignment between two regions [Fig. 19(b)]. Therefore, as it was shown by $\mathrm{Xu}$ et al. [156], it is expected that photo generated carriers move from the bilayer to the single layer region in the normal case. However, if the PTE is the dominant mechanism in photocurrent 
generation, the current would be directly proportional to the difference in thermoelectric power (S).

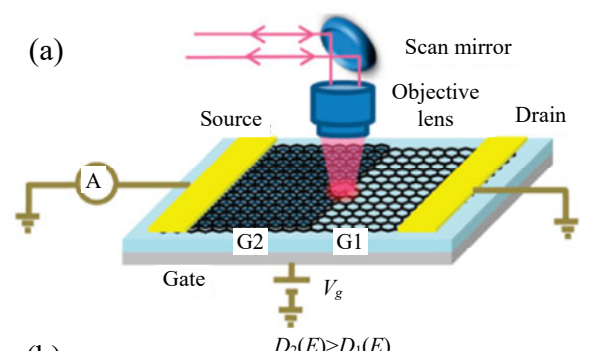

(b)

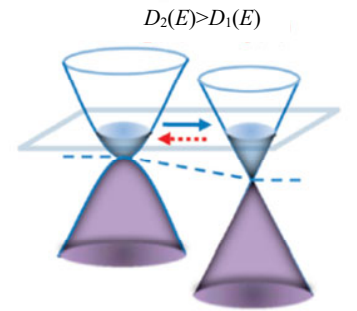

Fig. 11 Photo generated carriers movement between the bilayer and single layer graphene [156]: (a) schematics of an experimental setup with two regions of single and bilayer grapheme and (b) aligned Fermi level of bilayer (left) and single layer (right) graphene. The blue and red dashed arrows represent the electron flow direction induced by the built-in-electric field and by the thermo-electric effect, respectively.

The thermoelectric power itself is a measure of the partial molar entropy and according to the second law of thermodynamics; entropy is proportional to the DOS. Consequently, the hot carriers tend to diffuse to the material with larger $D O S$ to maximize the entropy. In the system of single and bilayer graphene $D O S_{2}>D O S_{1}$ thus the carriers should flow from the single to the bilayer which is in the opposite direction of photocurrent generated by built-in-electric field. These photogenerated hot carriers generated by PTE can produce a photovoltage which is related to thermoelectric power (seebeck coefficient) of each region $\left(S_{1}\right.$ and $\left.S_{2}\right)$ by $V_{\mathrm{PTE}}=\left(S_{2}-S_{1}\right) \Delta T$. It should be noted that in the graphene photodetectors of which PTE is the dominant mechanism, the device can achieve a high bandwidth compared with the photovoltaic based photodetectors, since in these systems instead of lattice heating, hot electrons generate the electronic response. The PTE effect is the dominate mechanism in graphene $\mathrm{p}-\mathrm{n}$ junctions $[151,157]$ or in suspended graphene [158] devices.

The bolometric effect is another photodetection mechanism which is based on the variation of local temperature of the graphene by incident radiation. When the electromagnetic radiation hits the graphene surface, it elevates the local temperature of the graphene by strong electron-phonon interaction, which in turn enhances the electron-acoustic phonon scattering. According to this temperature variation the resistance of the device increases and consequently it leads to a reduction in current. The thermal resistance $R_{h}=\mathrm{d} T / \mathrm{d} P$ (dP is the power of electromagnetic radiation) and the heat capacity $C_{h}$ are the key parameters in bolometric effect. The former defines the device's sensitivity and the latter determines the response time $\tau=R_{h} C_{h}$ of the device [159]. The low heat capacity implies the fast response in the devices and since graphene for a given area has low density of states, it has a fast response. Graphene also presents high bolometric sensitivity due to its high thermal resistance. There are two reasons for high $R_{h}$ in graphene. First, because of small Fermi surface, the cooling of electrons by acoustic phonons is inefficient; second, the cooling by optical phonons requires very high temperature $T_{e}\left(k_{B} T_{e}>0.2 \mathrm{eV}\right)$. In contrast to the PTE which is the dominate mechanism in p-n junctions, bolometric effect can appear in homogeneous graphene and it requires an external applied bias.

Photo-gating effect is another mechanism of photocurrent generation in the graphene devices which is based on modification of conductance $(\Delta \sigma=q u \Delta n)$ due to the variation of carrier density. This effect was first reported in the 1980s [160], however the "photo-gating" term was proposed by the group at Pennsylvania State University in 1992 [161] to the observation of anomalously high quantum efficiency values in the amorphous silicon $\mathrm{p}-\mathrm{i}-\mathrm{n}$ structure. The main difference between photogating and the bolometric effects is that the former is based on a light-induced change in carrier density (n), while the latter is based on the change in mobility $(\mu)$ due to the heating. The term gating refers to controlling one type of photo generated carriers. This can happen by trapping one type of generated carriers (electrons or holes) in the 
graphene by charge traps or in nearby molecules of nanoparticles. In another possibility, the nanoparticles, molecules or charge traps in the vicinity of graphene can act as the source of E-H pair generation and then one type of carriers' residue in resides in the particles, molecules or traps and another type transfer to the graphene by an internal electric field. Therefore, while the charges are transporting between the device contacts (source and drain), the residue charges gate the graphene sheet.

Another mechanism of photodetection, particularly in $\mathrm{THz}$ regime, was proposed by Dyakonov and Shur (DS) which is called by the same name or sometimes known as the plasma-wave-assisted photodetection mechanism $[162,163]$. The DS detection mechanism is based on the existence of a 2D electron gas in a field-effect transistor (FET) which can act as a cavity for plasma-waves. There are two distinguishable regimes of the photodetection known as resonant and non-resonant regimes of plasma-wave photodetection [163]. In the resonant regime, the plasma wave from source contact can reach to the drain in a time interval shorter than momentum relaxation time and due to the constructive interference of this plasma wave, the radiation can be detected. This constrictive interference resonantly enhances the response and can improve the signal up to 20 times compared with the broadband non-resonant signal. As a result, a DC-photovoltage component is generated between the contacts, which is a response to the incoming $\mathrm{THz}$ field, thus providing rectification of the signal. If the plasma waves over damp by impurities and phonon scattering, the launched plasma wave in the source decays before reaching the drain contact which is in the broadband non-resonant regime. Resonant detection of $\mathrm{THz}$ radiation in graphene FETs was theoretically discussed in [164] and it was concluded that in order for a device to operate in the $\mathrm{THz}$ spectrum, the typical linear device size $L$ is required to vary between $1 \mu \mathrm{m}$ and $10 \mu \mathrm{m}$. From the structural points of view, for generating $\mathrm{THz}$ photo voltage signal, graphene FET devices with broken-symmetry at the boundary or the contacts is required, however the over damped problem still persist [165]. A new structure based on large-area CVD-grown graphene sheets was reported by Yeung et al. [166] with the plasma wave resonance at $6 \mathrm{THz}$ and ability to tune far infrared or $\mathrm{THz}$ resonant detection. A new mechanism for sensitive response to $\mathrm{THz}$ radiation was also proposed due to the contributions of both plasma drag and convection effects [167]. Recently, various photodetection mechanisms in graphene photodetectors were introduced and structures proposed for the photodetection purpose were introduced.

\subsubsection{Metal-graphene-metal photodetectors}

Among various architectures proposed for photodetection, the metal-graphene-metal (MGM) photodetector is the simplest structure and the building block of other photodetectors, in which graphene is contacted with metal electrodes as source and drain which can be either of the same or two different metals [147, 154, 168-173]. The main parameter which induces the charge transfer in this class of devices is the work function difference between the metal pads and graphene. According to the work function difference between the metal pad and underneath graphene, the Fermi level of graphene in the region below the metal pads will be shifted [Fig. 12(a)]. Accordingly, because of charge transfer between the graphene and the metal contact a band bending is created in the graphene and due to pinning the graphene by top metal pad, a potential gradient extending $0.1 \mu \mathrm{m}-0.2 \mu \mathrm{m}$ in the metal-free graphene sheet appears [168]. Therefore, based on the metal contacts, $\mathrm{n}$ or $\mathrm{p}$ type doping can be formed which creates an in-homogenous doping profile at the junction along the channel.

If a back gate is introduced to the structure, the Fermi level of the graphene channel can also be controlled. When the junction of graphene/metal is illuminated by light, an E-H pair forms. The existence of the junction in the structure is crucially important in the photodetection process, as it creates 
an electric field which can separate the created E-H by light. As shown in Fig. 12(b), the electrostatic doping of graphene can be obtained by adding a top gate to the designed structure. The dual gate structure allows formation of nnn, ppp, npn, or pnp junctions, respectively [170]. The photovoltage in the contact region strongly depends on the incident light's wave length. Figure 13 shows the variation of photovoltage map for two excitation wavelengths of $457 \mathrm{~nm}$ [(Fig. 13(a)] and $785 \mathrm{~nm}$ [(Fig. 13(b)] in a pn-junction where the graphene channel is n-doped and under the metal contact it is p-doped. As it was shown for lower wavelength of $457 \mathrm{~nm}$, almost the entire metal contact area contributes to the photovoltage, with a maxima at the contact edge, while for a longer wavelength of $785 \mathrm{~nm}$, the photovoltage is limited close to the contact edge where the pn-junction was located, and vanishes where the graphene flake ends underneath the contact, indicated by the dotted line. This dependence on excitation wavelength is due to an increased absorption of the incident light at shorter wavelengths on the $\mathrm{Au}$ contact. This leads to a temperature rise on the metal contact, thus heating the pn-junction at the contact edge and producing a thermoelectric contribution to the photovoltage.

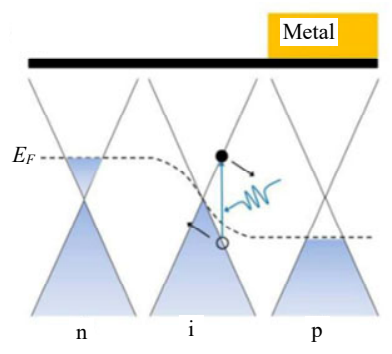

(a)

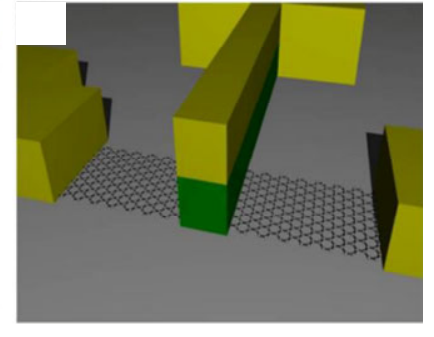

(b)
Fig. 12 Overview [170] of (a) energy-band profile in metal-graphene-metal photodetectors (MGM-PDs) and (b) transistor-like graphene-based PD employing a top gate.

This is a limitation for operation of photodetectors since in a large area of graphene sheet that most of the E-H pairs are generated, the effect of electric field is diminished and therefore the carrier recombines before they contribute to the external photocurrent. As it is already mentioned, this effect is more destructive in long-wavelength

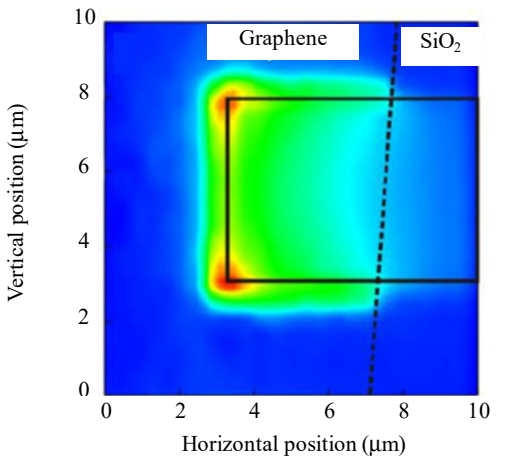

(a)

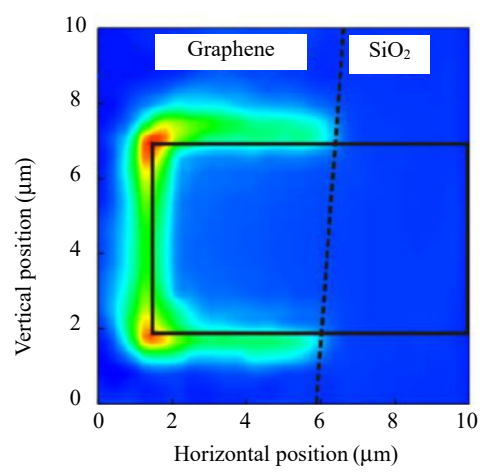

(b)

Fig. 13 Photovoltage maps [170] for (a) $457 \mathrm{~nm}$ and (b) $785 \mathrm{~nm}$ excitations.

regime. In order to solve this problem, interdigitated metal fingers and doping via electrostatic gating were proposed, which was designed to form high electric field in a large region of device. It has been shown that in the devices with symmetric metal contacts, the total photocurrent is zero [Fig. 14(a)], because the electric field in channel between neighboring fingers is symmetric $[147,168]$. In the case of graphene photodetectors with similar metal contacts, there is a limitation for applying a bias voltage since graphene is a semi-metal, and an external voltage would drive a large dark current through the device which not only restricts the voltage that can be applied, but also gives rise to strong shot noise. To overcome this drawback, interdigitated structures with asymmetric metal fingers, which have different work functions, have been proposed [Fig. 14(b)]. In this configuration, the field effectively separates the electrons and holes, and a photocurrent is produced when light is shone onto the device. The benefit of this configuration is that the resulting built-in field acts on the entire area 
of the device and there is no need to apply a bias voltage for the device to operate, which allows eliminating unwanted noise from the output of the device. Despite the advantages mentioned above, a serious drawback of this structure is that the actual amount of light absorption by graphene is reduced due to the presence of the electrodes.

The doping via electrostatic gating [169] is another approach which also has the same fundamental by MGM photodetector structures [Figs. 14(c) and 14(d)]. As it is already known, an electrostatic potential is constant over the edge of metal contacts. Therefore, the electric field is perpendicular to the edge of the electrode and hence, the photo carriers generated in graphene near an electrode contact will be pushed normal to the edge of the electrode, thereby gaining momentum along this direction. This initial momentum will soon be lost after many scattering events in other materials. However, because of a long carrier mean free path (as large as $0.4 \mu \mathrm{m}-0.6 \mu \mathrm{m}$ ) of graphene, the average momentum will still be along the initial direction in the graphene layer. This leads to maximum PC collection by the electrode positioned along this direction [147, 148]. The same drawback still persists since these devices also employ opaque metallic structures which would introduce significant haze caused by light scattering. In MGM structures in addition to the PV effect, the PTE

(a)

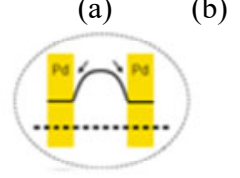

(b)

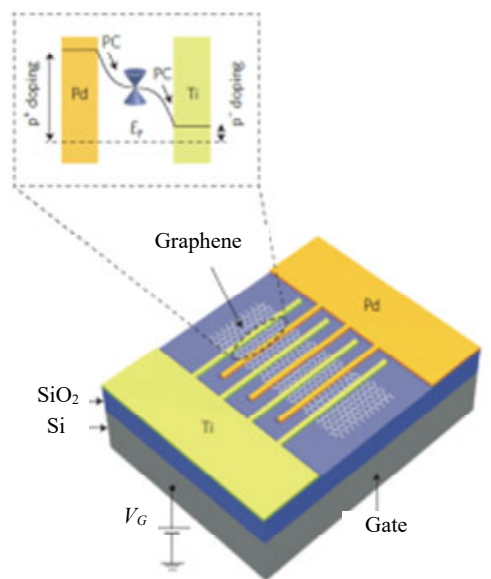

effect can also contribute to photocurrent $[174,175]$. In the following two cases, PTE is the dominate mechanism, first in the region near a $p-n$ junction and second, at the interface of single-layer and bi-layer graphene. The current generated by PV and PTE are in an opposite direction and can be distinguished from each other. The polarity of the photocurrent generated by PV is dependent on the direction of the built-in electric field. However, in the PTE model, the generated photocurrent is equal to thermal voltage divided by device resistance $R$. The thermal voltage is proportional to the local temperature increase in the junction, as well as to the difference in the Seebeck coefficients $(S)$ between graphene and the electrode. Subthreshold swing $(S)$ mainly depends on the threshold voltage $\left(V_{t}\right)$ and reversing sign as the majority carrier changes from electron to hole. When both photo voltaic effect (PVE) and PTE contributions have the same sign, a large photocurrent would be created. However, in the regime where the built-in electric field just switches the sign, the two contributions can have opposite signs and the photocurrent can then have either sign depending on their relative strengths. In bipolar p-n junctions and also unipolar $\mathrm{p}^{+} \mathrm{p}$ or $\mathrm{nn}^{-}$junctions, the PTE current shows sign reversal [157]. Together with the sign reversal when switching the electric field direction, this leads to a sixfold sign change in the photocurrent (Fig. 15).

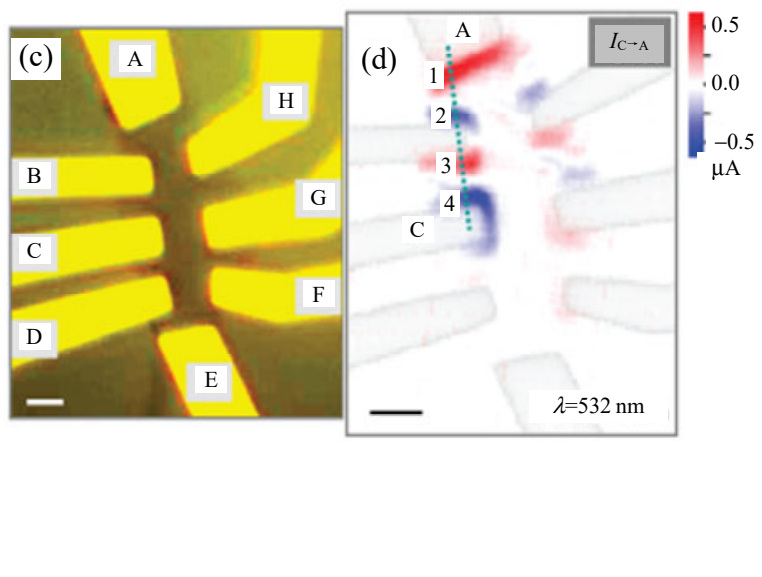

Fig. 14 Potential profile of symmetric and asymmetric metal-graphene-metal photodetector [169, 176]: (a) potential profile of a metal-graphene-metal photodetector with symmetric contacts, two contributions cancel each other and the total photocurrent is zero, (b) potential profile for an asymmetric device. For an appropriate gate bias, the photocurrent at both contact points in the same direction, and (c) optical image of the graphene device, and (d) combined light reflection and photocurrent image. 


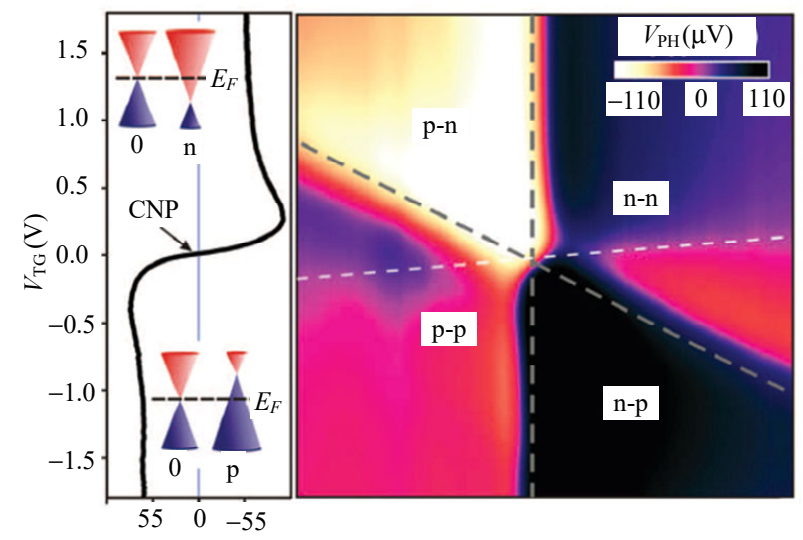

Fig. 15 Photovoltage as a function of back-gate voltage $\left(V_{\mathrm{BG}}\right)$ and top-gate voltage $\left(V_{\mathrm{TG}}\right)$; a sixfold change of polarity was observed. Grey dashed lines show high resistance as obtained from transport measurements [157].

\subsubsection{Graphene-semiconductor hetero-junction photodetector (GSHP)}

The GSHP is another class of photodetectors, which is designed to solve a few of fundamental problems in application of graphene in photodetectors which are the weak absorption $(A \approx 2.3 \%)$, the short lifetime of the order of picoseconds of photogenerated carriers in graphene $[177,178]$, and the small effective photodetection area. GSHP are basically reversed biased G-S Schottky diodes. A comprehensive review of the G-S Schottky diodes graphene was recently published by Bartolomeo [179]. In GSHP, the depletion layer of semiconductor absorbs the incident light and the separation and transport of photogenerated carriers happening in this region, while the effective photodetection area is restricted by the G-S contact area. These photogenerated carriers can contribute to the photocurrent, if they reach to the graphene without the issue of the fast e-h pair recombination in graphene. Another benefit of GSHP is highly external quantum efficiency which is in the range of $50 \%$ to $65 \%$, giant responsivity because of intrinsic gain mechanism and the ability to tune the responsivity by the applied reverse voltage bias which makes it suitable for application in fast and sensitive photodetection at variable brightness. Figure 16(a) shows a CVD-grown SLG on lightly doped n-Si proposed by An et al. [180]. They have measured the Schottky barrier heights of the device in the dark condition, and shown that it was pinned to the charge neutrality level of its own surface states, with a Schottky barrier height $\varphi_{B} \approx 0.8 \mathrm{eV}$ [Fig. 16(b)]. As shown in Fig. 16(c), under conditions of no bias and low power $(P=1.23 \mu \mathrm{W}$ and $\lambda=488 \mathrm{~nm})$, the current-voltage characteristics of the device follow conventional rectifying and photodiode-like behavior, respectively. In graphene a quasi Fermi level can be formed due to the built-in electric field in the junction with Si. Parameters such as initial position of graphene Fermi level and number of injected holes from $\mathrm{Si}$ determine the position of this quasi Fermi level. At low incident power a conventional photodiode-like response is observed, since due to the configuration of Fermi energy level the photoexcited holes can all find accessible states in graphene to inject in. In high incident powers (up to $P=6.5 \mathrm{~mW}$ ), since there are not enough states in graphene for the photogenerated hole injection, the $I-V$ behavior deviates from that of a conventional photodiode. At forward bias [Fig. 16(d)], the number of available states for the photoexcited holes to inject into graphene from $\mathrm{Si}$ is very small, therefore increasing incident power could not change the current and consequently the forward $I-V$ characteristics remain unchanged.

On the other hand, a reverse bias on graphene modifies the $E_{F}$ and creates a large number of available states for the carriers to inject into [Fig. 16(e)] and allows the collection of all the injected holes. As a result, the photocurrent can completely recover under small reverse biases. The proposed device demonstrated high photovoltage responsivity exceeding $10^{+7} \mathrm{~V} / \mathrm{W}$ at low power $(\sim 10 \mathrm{nW})$ which was much higher than that reported in MGM photodetectors with responsivity of $1 \mathrm{~A} / \mathrm{W}-2 \times 10^{-2} \mathrm{~A} / \mathrm{W}[181,182]$. 
(a)

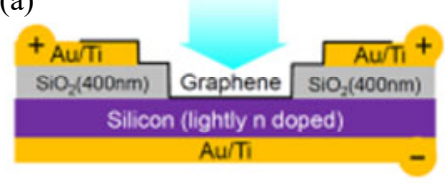

(d)

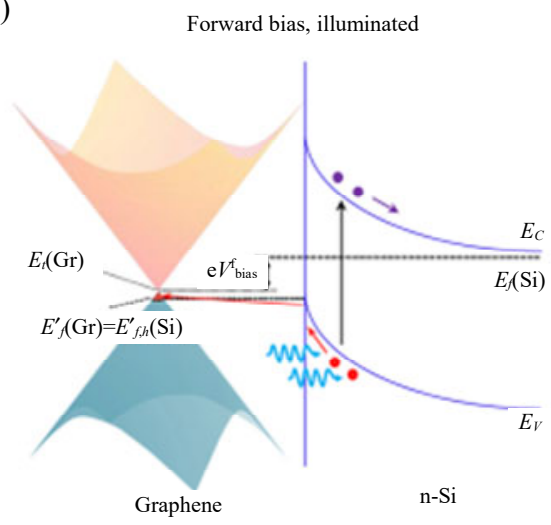

(b)

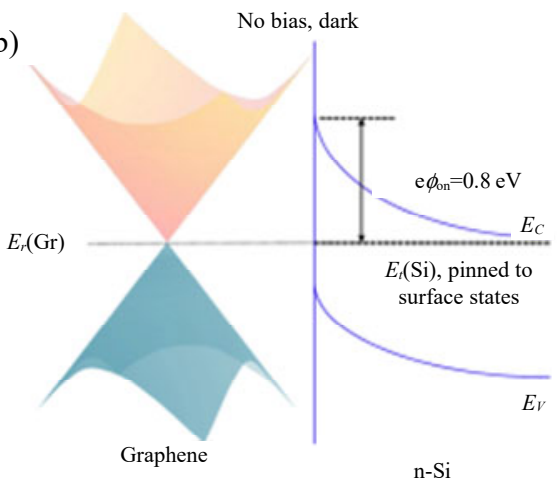

(c)

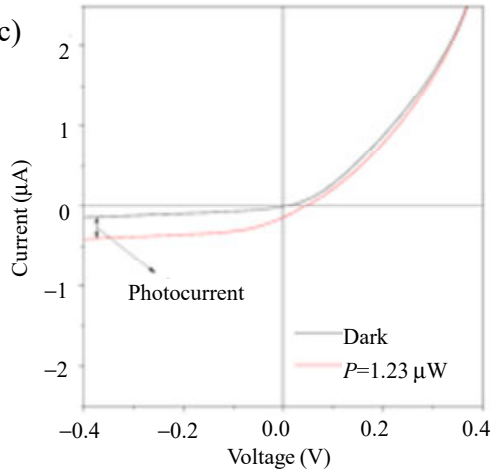

(e)

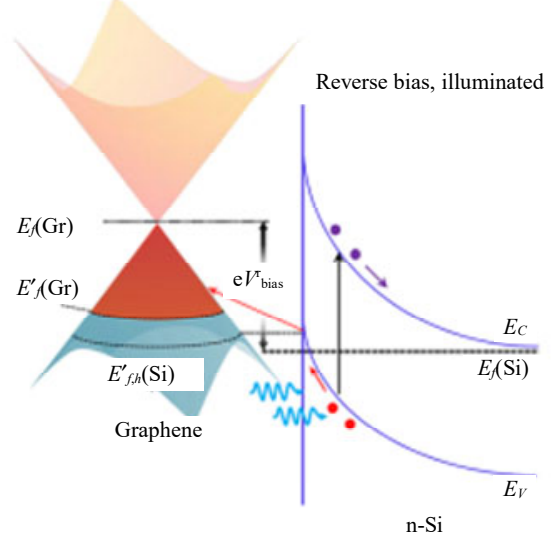

Fig. 16 Graphene-semiconductor hetero- junction photodetector (GSHP) [180]: (a) a side view of monolayer graphene/Si hetero-junction device, (b) energy band diagram at the thermal equilibrium in darkness, (c) current-voltage $(I-V)$ characteristics of device under darkness and weak illumination $(P=1.23 \mathrm{~W}$ and $\lambda=488 \mathrm{~nm})$, (d) application of a forward bias $\left(V_{f}\right.$ bias) resulting in the strongly suppressed photocurrent, and (e) application of a reverse bias ( $V_{r}$ bias). The external bias controls the position of the Fermi level and consequently the number of photo-excited carriers that can inject from Si (i.e., the photocurrent).

\subsubsection{Hybrid photodetectors}

Hybrid photodetectors are another category of photodetectors which have been designed to improve the sensitivity of graphene photodetectors for detection of low intensity incident photons even at single photon level. The device is principally a back gate phototransistors modified by appropriate sensitizing centers for absorbing incident light and transfer of photogenerated carriers to the graphene layer by photo-gating mechanism. Implementation of colloidal quantum dots (CQDs) and biomolecules [183-187], modification of ligands at the quantum dot (QD) surface [188-190], and graphene composites consisting of graphene and graphene quantum dots on a rigid and stretchable substrate $[191,192]$ have been used as sensitizing material in hybrid structures for obtaining an ultra-high sensitive photodetector. CQDs made of $\mathrm{PbS}, \mathrm{CdS}$,
$\mathrm{ZnO}$, and $\mathrm{TiO}_{2}$ can be used as light-absorbing particles to sensitize graphene. For instance, $\mathrm{ZnO}$ CQDs have been used because of their large bandgap (about $3.3 \mathrm{eV}$ ), which allow to focus on the device response to UV light and screen the influence from visible and infrared light source [190, 193-195]. Figure 17(a) presents a typical $\mathrm{ZnO}$ CQDs/graphene hybrid structure used as photodetectors. The main parameter that causes $\mathrm{ZnO}$ QDs to help in faster response of graphene was the electron transfer between the QDs and graphene with assistance from the oxygen molecules in air. The proposed charge transfer mechanism is schematically presented in Figs.26(b) to 26(f). For this structure, photo conductive gain as high as $10^{7}$ has been reported, which can be utilized for practical graphene based photodetectors with very high responsivity. When the structure is illuminated by photons with energy larger than band gap, E-H pairs 
are generated [Fig. 17(b)] and soon separated, with the high density of available holes trapped at the surface along the potential slope, leaving behind unpaired electrons [Fig. 17(c)]. The holes then discharge the adsorb oxygen ions and the neutral oxygen molecules are desorbed from the surface

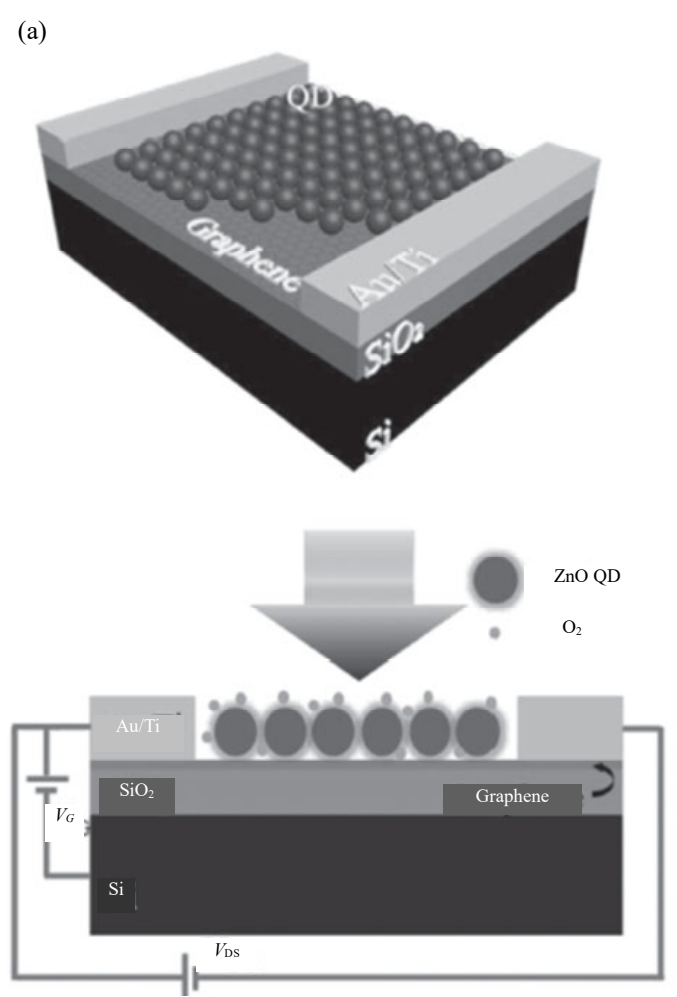

[Figs. 17(d) and 17(e)]. The unpaired electrons are transferred to the graphene layer and pull up the Fermi energy level, and consequently the charge neutrality point of graphene is left-shift [Fig. 17(f)]. The passive sensitizing layer of CQDs can also be replaced by an active structure.
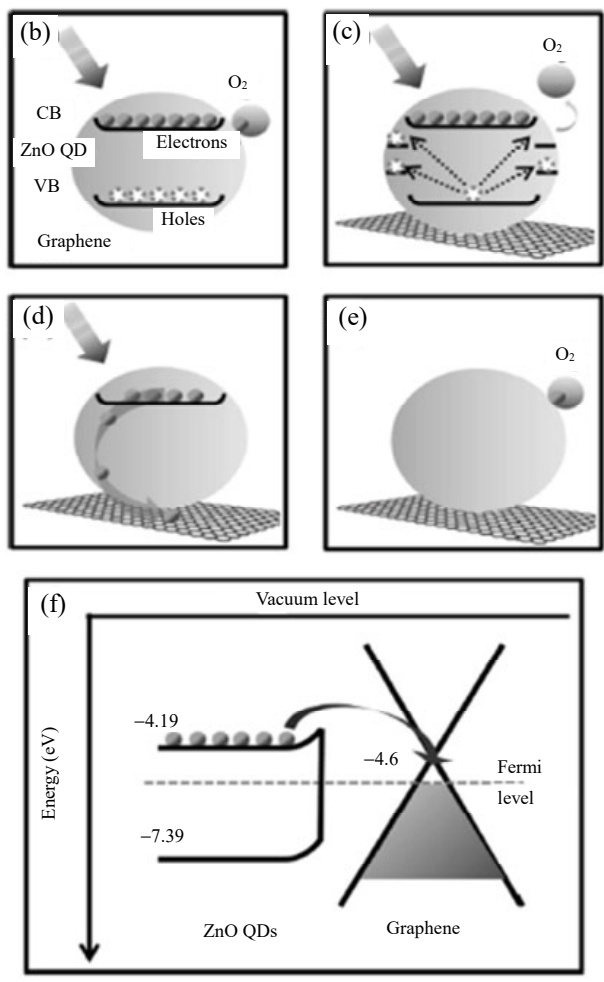

Fig. 17 Hybrid photodetector [191]: (a) 3D schematic view of the graphene device coated with ZnO QDs and the corresponding side view of the device with the presentation of the oxygen-assisted charge transfer process, (b) electron-Hole pairs generation, (c) trapped holes at the surface states leads to the formation of unpaired electrons through discharging the oxygen ions on the surface which cause desorption of the oxygen molecules, (d) the electrons movement from the QDs to the graphene, (e) no more electron-hole pairs are generated when laser turned off, and (f) the energy diagram of ZnO QDs and graphene.

This is performed by integrating a colloidal quantum dot photodiode atop of a graphene phototransistor in order to form a hybrid photodetector device [183]. The new hybrid detector improves the performance in terms of speed, quantum efficiency and linear dynamic range (LDR). A quantum efficiencies in excess of 70\% (QE 25\% in passive $\mathrm{PbS}$ and $\mathrm{ZnO}$ ), gain of $10^{5}$, LDR of $110 \mathrm{~dB}$ and bandwidth of $1.5 \mathrm{kHz}$ have been measured which is among the highest performance up to date.

\subsubsection{Integration with planar photonic crystal cavities, wave guides and plasmonics}

Another widely used approach for fabrication and improving the performance of photodetectors is integration of graphene into an optical microcavity or planar photonic crystal cavities, wave guides, and plasmonics [196-198]. It is known that, the SLG present non-resonant absorption of just $2.3 \%$ of incident light and optical microcavity is an effective way to improve the photo-responsivity of the devices. In [182], two distributed Bragg mirrors implemented as reflectors, consisting of quarter-wavelength thick layers of alternating materials with varying refractive indices, form a high-finesse planar cavity [Fig. 18 (a)]. The graphene layer is then sandwiched between these 
reflectors. With this configuration, a responsivity of $S=21 \mathrm{~mA} / \mathrm{W}$, an enhancement of 26 times in absorption as compared with the absorption of free-standing graphene has been reported. Incident light-graphene interaction can also be enhanced by using light captured in a sub-wavelength nano-cavity in a planar photonic crystal (PPC) [199]. (a)

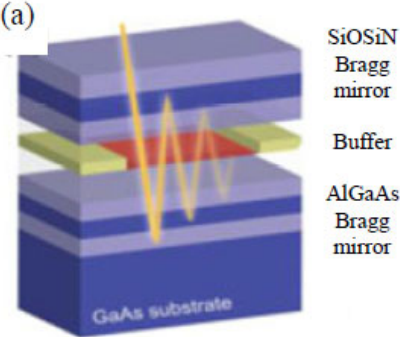

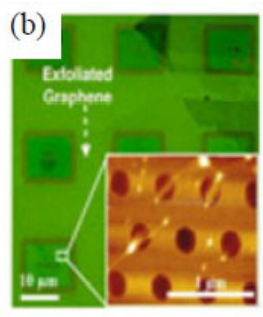

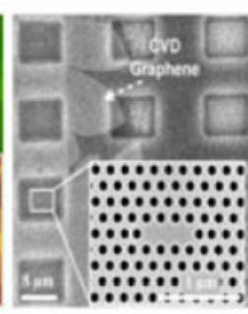

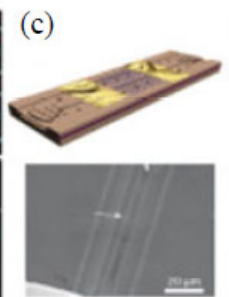

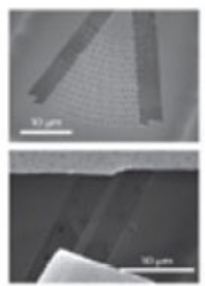

Fig. 18 Integration of graphene into an optical microcavity or planar photonic crystal cavities, wave guides, and plasmonics [182, 198]: (a) schematic drawing of a graphene micro-cavity photodetector, (b) planar photonic crystal cavities integrated with graphene, and (c) schematic of graphene/silicon-heterostructure waveguide photodetector.

Figure 18(b) shows an optical microscope image of the PPC cavities integrated with exfoliated graphene. It is found that the PPC reduces the cavity reflection by more than 100 times, which can improve the absorption of grapheme-cavity system more than $45 \%$. Integrating graphene with plasmonic nanostructures is another useful technique to overcome the limitation of low responsivity in graphene photodetectors. Surface-enhanced Raman spectroscopy of graphene was the initial application of plasmonic nanostructures [200]. Figure 18(c) indicates a graphene/silicon waveguide photodetector on SOI that operates from the visible to mid-infrared spectral range [201]. The device consists of a monolayer graphene which is transferred deterministically to the waveguide. The suspended membrane waveguide, which has been fabricated by electron-beam lithography (EBL) and dry reactive ion etching, is critical in the design to avoid absorption of mid-infrared light by the buried oxide (BOX) $\left(100 \mathrm{~dB} \cdot \mathrm{cm}^{-1}\right.$ at $\left.2.75 \mathrm{~mm}\right)$ and also to take full advantages of the transparent wavelength region of silicon, which covers the range between $1.2 \mathrm{~m}$ and $8.0 \mathrm{~m}$. The reported responsivity of this device was $10^{-4} \mathrm{~A} / \mathrm{W}$, however the responsivity of $0.05 \mathrm{~A} / \mathrm{W}-0.13 \mathrm{~A} / \mathrm{W}$ was also reported in $[199,201$, 202] which is compatible with the state-of-the-art photodetectors made of GeSn [203]. Therefore, it makes the design a promising candidate for Si-compatible photodetector for long-wavelength ( $\mathrm{L}$ and U) bands. More recently, a graphene photodetector with a bandwidth of more than $76 \mathrm{GHz}$ has been fabricated on 6 inch silicon-on-insulator (SOI) wafers in a semiconductor pilot line [204]. The device has been fabricated by placing a graphene layer on the top of the waveguide within the evanescent field that surrounds the waveguide. The device takes the advantages of the fact that for generating the photo current the bolometric effect of the entire graphene channel contributes and circumvents the fabrication constraint related to an exact positioning of a defined doping gradient. In the low frequency regime, the reported sensitivity for the device was $0.13 \mathrm{~V} / \mathrm{W}$. While in the high frequency regime, the $3 \mathrm{~dB}$ bandwidth for the device was $67 \mathrm{GHz}$ and a dependence of the bandwidth on the bias voltage could not be observed. The pros and cons of mentioned graphene based photodetectors have been summarized in Table 1. According to the above discussions, in MGM based structures, devices suffer from low light absorption, difficulty of extracting photoelectrons (only a small area of the $\mathrm{p}-\mathrm{n}$ junction contributes to current generation), absence of a photocurrent for the uniform flood illumination on both contacts of the device, and 
existence of opaque metallic nanostructures [205, 206]. As an alternative, full graphene photodetectors was utilized to overcome some of the fundamental problems associated with the graphene based photodetectors.

Table 1. Pros and cons of common graphene based photodetectors.

\begin{tabular}{|c|c|c|}
\hline Photodetector & Pros & Cons \\
\hline$M G M$ & Simple structure & $\begin{array}{l}\text { 1. Carriers recombine } \\
\text { before they contribute to } \\
\text { the external photocurrent } \\
\text { 2. Large dark current } \\
\text { 3. Strong shot noise } \\
\text { 4. Presence of the opaque } \\
\text { electrodes }\end{array}$ \\
\hline GSHP & $\begin{array}{l}\text { 1. Strong absorption } \\
\text { 2. Long photogenerated } \\
\text { carriers lifetime } \\
\text { 3. Large effective } \\
\text { photodetection area } \\
\text { 4. High external quantum } \\
\text { efficiency } \\
\text { 5. Giant responsivity } \\
\text { 6. Fast carrier separation }\end{array}$ & $\begin{array}{l}\text { Relatively-high density of } \\
\text { surface states limit the } \\
\text { specific detectivity }\end{array}$ \\
\hline $\begin{array}{c}\text { Hybrid } \\
\text { photodetectors }\end{array}$ & $\begin{array}{l}\text { 1. Low intensity incident } \\
\text { photon detection } \\
\text { 2. High speed, } \\
\text { 3. High quantum efficiency } \\
\text { 4. High linear dynamic } \\
\text { range (LDR) }\end{array}$ & $\begin{array}{l}\text { Complicated } \\
\text { fabrication }\end{array}$ \\
\hline $\begin{array}{l}\text { Integration with } \\
\text { planar photonic } \\
\text { crystal cavities, } \\
\text { wave guides and } \\
\text { plasmonics }\end{array}$ & $\begin{array}{l}\text { 1. High responsivity } \\
\text { 2. High external QE } \\
\text { 3. Low dark current shot } \\
\text { noise } \\
\begin{array}{l}\text { 4. Si-compatible PD for } \\
\text { long-wavelength (L and U) } \\
\text { bands }\end{array}\end{array}$ & $\begin{array}{l}\text { 1. Complicated device } \\
\text { fabrication } \\
\text { 2. Expensive }\end{array}$ \\
\hline
\end{tabular}

\section{Full graphene photodetectors}

Full graphene photodetectors (FGPDs) with simple structures are suitable candidate for flexible, ultra broadband, and fast photodetection since its absorption spectrum covers the entire UV to terahertz wavelengths and the carriers move with ultra-fast mobility in the graphene structure. These unique properties make FGPDs an alternative to overcome some one the main obstacles of conventional broadband photodetectors which generally have a complicated system configuration with high cost, consisting of trichroic prisms, optical filters, charge coupled devices, etc. [207]. In the following section, we overviewed the early FGPDs and their limitations as well as innovative approaches which have been applied to improve the operational performance of FGPDs. One of the first full-graphene photodetector structures was proposed and fabricated by Withers et al. [208]. The device's transparency enabled the development of a new generation of transparent photodetectors which had not been suffered from haze. The proposed structure is indicated in Fig. 19(a). The active part of the device consists of a $\mathrm{FeCl}_{3}$ intercalated few layer graphene $\left(\mathrm{FeCl}_{3} / \mathrm{FLG}\right)$ which is also known as dubbed graphexeter and a pristine graphene on a $\mathrm{Si} / \mathrm{SiO}_{2}$ substrate. The intercalation of FLG performed by two-zone vapor transport technique [209, 210], however other techniques for intercalation of graphene could also been applied $[30,211,212]$. The intercalation of $\mathrm{FeCl}_{3}$ in graphene known to p-type dopes the graphene and increases the charge carrier density of graphene up to $9 \times 10^{14} \mathrm{~cm}^{-2}$. It also reduces the room temperature square resistance of graphene to just a few ohm [213]. The Raman analysis revealed that due to selection of tri-layers structure in this experiment, the final structure after intercalation is a layer of $\mathrm{FeCl}_{3}$ sandwiched between a graphene monolayer and a graphene bilayer. After the intercalation process, the pristine graphene is transferred on the graphexeter by using the PMMA polymer assisted transfer technique $[97,109,111]$. Then the independent metal contacts formed on the bottom $\mathrm{FeCl}_{3} / \mathrm{FLG}$ and pristine graphene layer to characterize the device. In order to investigate the performance of fabricated photodetector, the active region of device has been illuminated by a $532 \mathrm{~nm}$ He-Ne laser focused to $1.5 \mu \mathrm{m}$ spot size at a power of $8.2 \mu \mathrm{W}$. The beam is chopped at $370 \mathrm{~Hz}$, and the chopper is used as reference to a lock-in amplifier which measures the photovoltage. In order to 
understand the mechanism of photovoltage formation a global back gate on heavily doped underneath, $\mathrm{Si}$ has been designed by tuning the chemical potential of graphene. The photovolatge generated in the device has been measured by scanning the active part of the photodetector by laser spot presented in Fig. 19(b). The maximum photovoltage has been generated at the Au/FLG (blue) and $\mathrm{FLG} / \mathrm{FeCl}_{3}$ FLG (red) interfaces, while the photovoltage at the $\mathrm{FeCl}_{3} \mathrm{FLG}_{\mathrm{F}} \mathrm{Au}$ is nearly zero. In order to understand the origin of the photovoltage generated in the device, they modulated the chemical potential in the pristine FLG through variation of back gate voltage. As shown in Fig. 19(c), the generated photovoltage strongly depends on the interface that was measured. A photovoltage of up to $\pm 30 \mathrm{mV} / \mathrm{W}$ was measured for $\mathrm{Au} / \mathrm{FLG}$ (blue) and $\mathrm{FLG} / \mathrm{FeCl}_{3}$ FLG (red), while the value measured for $\mathrm{Au} / \mathrm{FeCl}_{3}$ FLG was almost zero. The switching of photovoltage sign was related to the movement of Fermi energy across the charge neutrality point by gate voltage. More interestingly, it was reported that the photovoltage measured at the $\mathrm{FLG} / \mathrm{FeCl}_{3}$ FLG interface was in the same order of magnitude with that generated at the Au/FLG and also equivalent or larger than that previously reported in doubly gated graphene p-n junctions. This observation confirmed that the origin of photovoltage generated in the photodetector was the photo-thermo-electric (PTE) effect. The PTE has been already discussed in detail in Section 5. When the carriers excite from valence band to the conduction band by incident photon, a hot fermion distribution is formed by the carriers relax back to the Fermi level. Then, due to the existence of a photoactive interface as a result of difference in density of states, the carriers diffuse from one side to the other side of the interface, leading to a photovoltage. The measured photovoltage also confirmed that the charge transfer could not be responsible for the measured photovoltage, since there was a decrease in the photovoltage for high doping of the non-intercalated flake. It should be noted that in the proposed design by considering chromium, the effect of contacts in photovoltaic effects was suppressed since it induced a very small band bending in graphene.

One of the limitations in graphene photodetectors is the low value of linear dynamic range (LDR) which is due to the intrinsic hot-carrier dynamics, and causes deviation from a linear photoresponse. The multiplication of hot carriers which limits the implementation of graphene in high-resolution applications is another point which should be considered. In order to solve these issues, Adolfo et al. [214] fabricated a device which the starting material was a four-layer $\mathrm{FLG} / \mathrm{FeCl}_{3}$ and $\mathrm{FeCl}_{3}$ was introduced only among the top three carbon layers. The $\mathrm{FLG} / \mathrm{FeCl}_{3}$ exposed to 532-nm laser light with the power of $15.3 \mathrm{MW} / \mathrm{cm}^{2}$ for $3 \mathrm{~s}$. They have reported that the laser radiation could locally tailor $\mathrm{FeCl}_{3}$ intercalation in graphene. The region beyond the laser spot remained unchanged, therefore the resolution of the $\mathrm{FeCl}_{3}$ displacement was defined by the laser spot profile. This technique can be used similar to the photolithography, for locally customize the chemical functionalization of graphene layers. In the region of laser irradiated, a reduction of $-0.6 \times 10^{14}$ in hole concentration was measured which defined an abrupt $p$ - $\mathrm{p}^{\prime}$ junctions at the boundaries of the laser exposed region. The origin of photocurrent at $\mathrm{p}-\mathrm{p}^{\prime}$ junctions of $\mathrm{FeCl}_{3}$-FLG was shown to be pure PV. The NEP of the photodetector was measured to be $4 \mathrm{~kW} / \mathrm{cm}^{2}$, thus an LDR of $44 \mathrm{~dB}$ has been obtained in the device which was 4500 times larger than that of previously reported graphene photodetectors $(\mathrm{LDR} \approx 7.5 \mathrm{~dB})[154]$ and $\sim 800$ times larger than that of other functionalized graphene devices (LDR $\approx 15 \mathrm{~dB})$ [215]. 


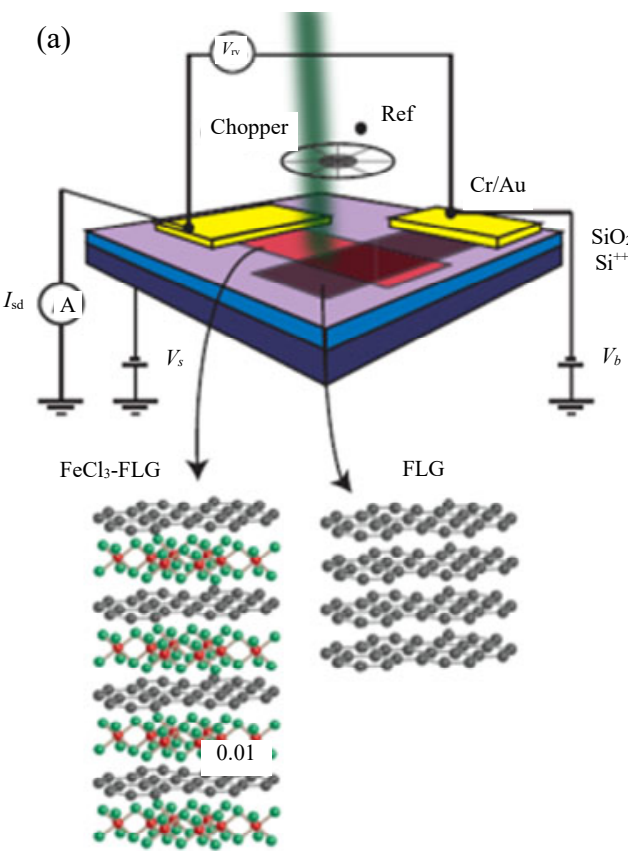

(b)

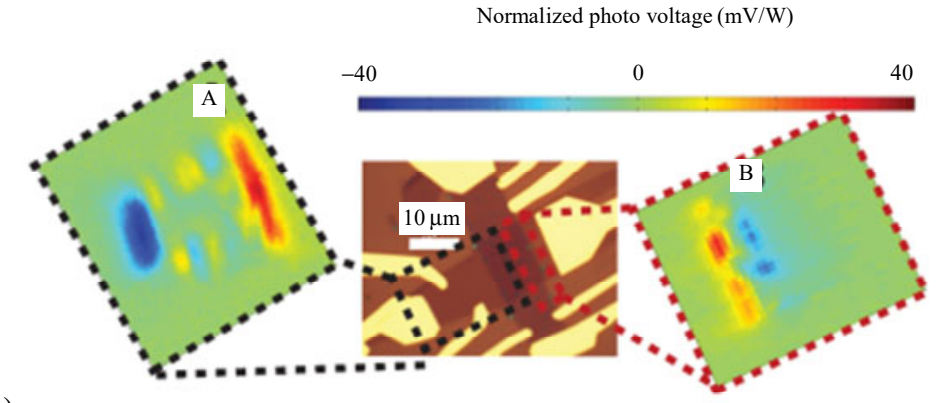

(c)
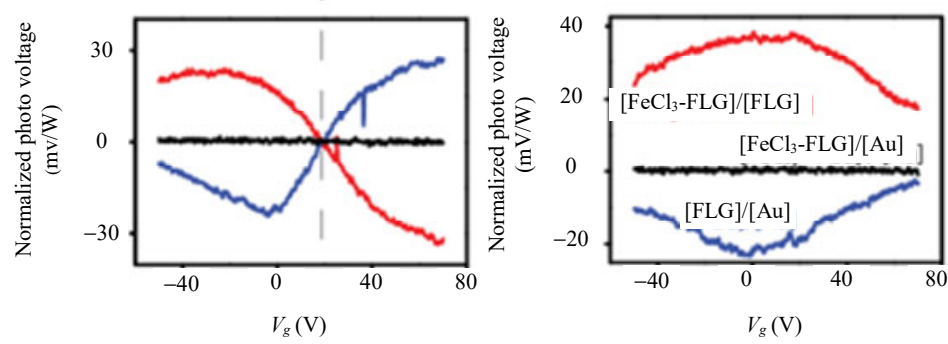

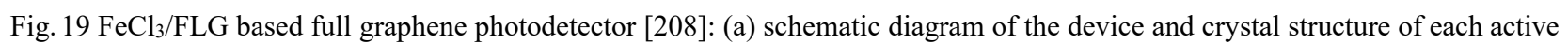
parts of the device which consists of FLG flakes and fully intercalated $\mathrm{FeCl}_{3} / \mathrm{FLG}$; (b) color-coded photovoltage spectroscopy for the interfaces of the device indicated in the optical micrograph picture. The blue region in the photovoltage maps at the Au/FLG interface, and the red is the $\mathrm{FLG} / \mathrm{FeCl}_{3}-\mathrm{FLG}$ interface; (c) the back-gate dependence of the photovoltage when the laser position is located at the $\mathrm{Au} / \mathrm{FeCl}_{3}$-FLG (blue), $\mathrm{FLG} / \mathrm{FeCl}_{3}-\mathrm{FLG}$ (red), and the $\mathrm{FeCl}_{3}-\mathrm{FLG} / \mathrm{Au}$ (black) for the interfaces $\mathrm{A}$ and $\mathrm{B}$.

$\mathrm{ABA} / \mathrm{ABC}$ stacking domain junctions in tri-layer graphene is another FGPD design proposed for photocurrent generation [216]. Figures 20(a) and 20 (b) present the stacking structures of 3LG with $\mathrm{ABA}$ and $\mathrm{ABC}$ stacking sequences [217]. A Bernal (ABA) structure is the most stable 3LG where the first two layers show honeycomb lattice stacking with the A-atoms in the second layer right above the A-atoms in the first layer and the B-atoms in the second layer are above the centers of the hexagons of the first layer, while the A and B carbon atoms in the third layer occupy exactly the same in-plane locations as the atoms in the first layer, as shown in Fig. 20(a). On the other hand, a meta-stable phase of $3 \mathrm{LG}$ exists with a rhombohedral (ABC) structure. In this structure, the carbon atoms in the third layer are located in such a way that the pairs of A-atoms in the first two layers are located over the centers of the hexagons of the third layer, as shown in Fig. 20(b). The main idea behind the proposed photodetector was that the junction between $\mathrm{ABA}$ and $\mathrm{ABC}$ stacked graphene with the same thickness would behave like a hetero-junction between two different semiconductors, and so photocurrent generation can be expected. A schematic diagram of a fabricated $\mathrm{ABA} / \mathrm{ABC}$ photodetectors is presented in Fig. 20(c). The photocurrent images with corresponding Raman images for the three devices which are measured simultaneously to correlate the photocurrent with the exact location of the ABA/ABC junction are shown in Fig. 20(d).

As it is shown that the ABA/ABC junctions in the graphene channel is the area that photocurrent is mostly generated. By analyzing the measured results with calculated parameters for obtaining the origin of the photo current, it was concluded that the Seebeck coefficient difference, i.e., the photothermoelectric effect was the dominant mechanism for the photocurrent in the ABA/ABC junction. The band alignment and the formation of built-in band offsets at $\mathrm{ABA} / \mathrm{ABC}$ junction also have been shown in Fig. 20(e). This offset forms as a result of shift in Dirac point energies of each section with respect to their vacuum values and significantly modifies back gate dependence of the Seebeck coefficient 
difference and Fermi energy difference. Because of the increased optical absorption of TLG, the responsivity of the ABA/ABC TLG photodetectors should be larger than that of metal-SLG devices. The reported maximum responsivity in $\mathrm{ABA} / \mathrm{ABC}$

(a)

(b)
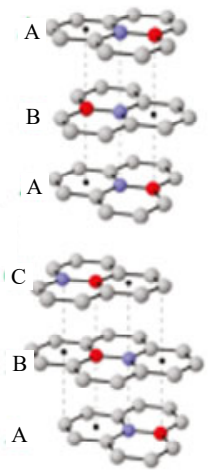

(c)

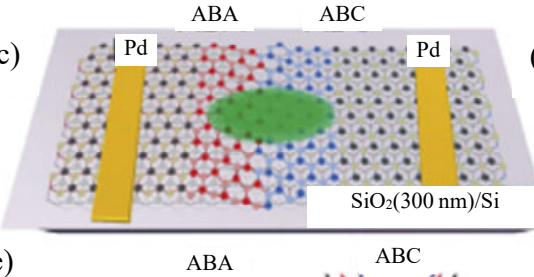

(e)

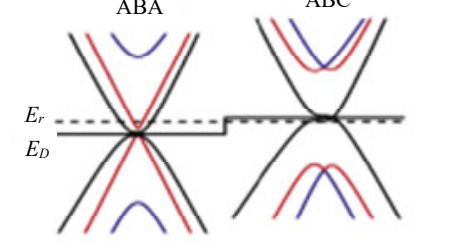

TLG device was $0.18 \mathrm{~A} / \mathrm{W}$. This value might be lower than the responsivity of the hybrid photodetectors, however it is comparable with other reported pure graphene photodetectors in the literature [149].

(d)

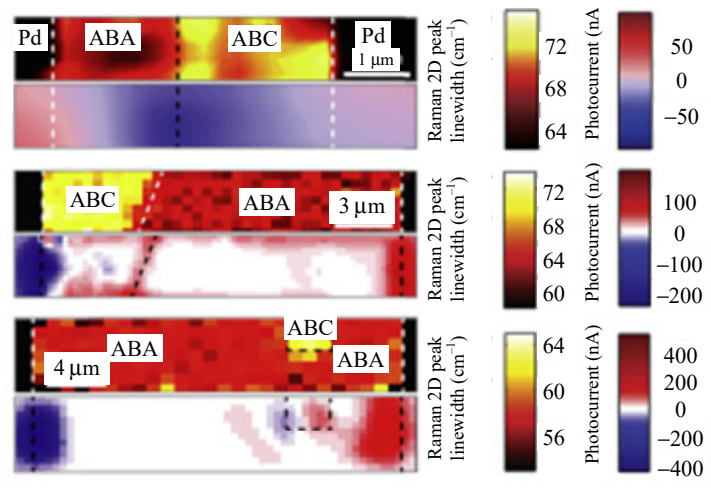

Fig. 20 Stacking structures of 3LG [216] with (a) ABA and (b) ABC stacking sequences, (c) schematic of a graphene photodetector with a lateral junction between $\mathrm{ABA}$ - and $\mathrm{ABC}$-stacked domains in 3LG (ABA/ABC photodetector), (d) comparison of 2D Raman peak line width (top) and photocurrent (bottom) images of 3 different $\mathrm{ABA} / \mathrm{ABC}$ photodetectors, and (e) band alignment of the $\mathrm{ABA} / \mathrm{ABC}$ junction in $3 \mathrm{LG}$ for $V_{G}=V_{\mathrm{CNP}}$.

In most of the FGPDs, the photoresponsivity is very low (a few $\mathrm{mA} / \mathrm{W}$ ), originating from the absorption of thin graphene layer $(\sim 2.3 \%)$, the small effective detection area of graphene, and the short photo-generated carrier lifetime due to the gapless energy bands. In order to improve the responsivity structures such as graphene field-effect transistor (FET) on a lightly p-doped $\left(\mathrm{Si} / \mathrm{SiO}_{2}\right)$ substrate, graphene decorated by graphene quantum dots (QDs) [218] have been proposed. Here, we discuss the latest one whose configuration is shown in Fig. 21(a). The device consists of a graphene layer, graphene quantum dots (QDs), and carbon-based conductive pastes. The main idea behind application of graphene QDs is their size-tunable optical response, efficient multiple carrier generation, non-toxicity, superiority of a large optical absorptivity, and low cost [219-221]. Moreover, it is known that carbon-based conductive pastes show preferable properties compared with other metal contacts for electrically contacting carbon nanostructures [222, 223]. Therefore, by integrating zero-dimensional and 2D grapheme, a novel all carbon-based photodetector has been fabricated. This device takes advantages of a large absorption coefficient of graphite QDs and a high conduction path provided by graphene. The device also exhibits an ultrahigh responsivity in the order of $4 \times 10^{7} \mathrm{~A} / \mathrm{W}$ which can be understood by the analysis of band alignment across the interface between graphite QDs and graphene.

In Fig. 21(b), the transmission electron microscopy (TEM) image of the synthesized graphite QDs on the surface of graphene film with an average diameter of $6 \mathrm{~nm}$ and relatively homo-dispersed distribution with a density of particle number of $2 \times 10^{11} \mathrm{~cm}^{-2}$ on the graphene device is presented. Figure 21(c) presents the photocurrent and the responsivity measurements as a function of the $V_{\mathrm{DS}}$ under illumination of a $\mathrm{He}-\mathrm{Cd}$ laser with different excitation intensities. The improvement of performance in the device with QDs could be clearly observed. According to the variation of photocurrent it can be concluded that the carriers in the graphene sheet magnified due to the charge transfer arising from the optically excited graphite QDs. The mechanism of photo sensing was examined by fabrication of a back gate graphite quantum dots/graphene phototransistor. In Fig. 21 (d), the band alignment between the graphite QDs and 2D graphene films is shown. It is known that the work functions of the highest occupied states and the lowest unoccupied states of the graphite QDs are 
estimated to be $6.14 \mathrm{eV}$ and $2.7 \mathrm{eV}$, respectively and the energy level of the Dirac point of intrinsic graphene film is at about $4.5 \mathrm{eV}$. Since graphite QDs are found to be of p-type nature, the corresponding Fermi energy is close to its highest occupied states. Therefore, as it is also shown in the band diagram, the photogenerated holes in graphite QDs will transfer to the graphene sheet which induces a p-type doping effect in graphene and contributes to the photocurrent. This also leads to the shift of the Dirac point from a lower voltage to a higher voltage. Such carrier transfer has known to allow a more effective charge separation in graphite QDs and consequently inhibits the E-H recombination [225, 226]. Accordingly, a fast increase in the photocurrent under light illumination and a slow decay after turning off the light had been observed because of the fast carrier transfer process from graphite QD to graphene and the high carrier mobility of graphene sheet and the spatial separation of electrons and holes, which defers the electron-recombination, respectively. In a similar work, Yu et al. [224] reported fabrication of a

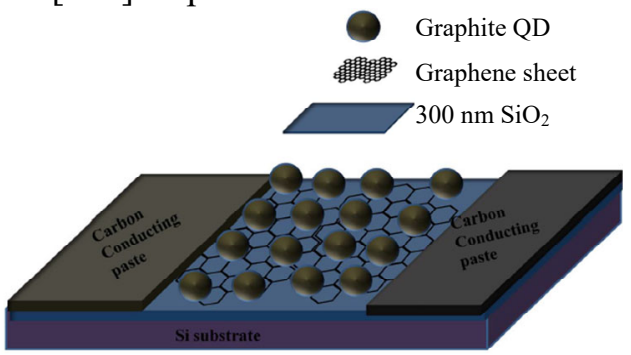

(a)
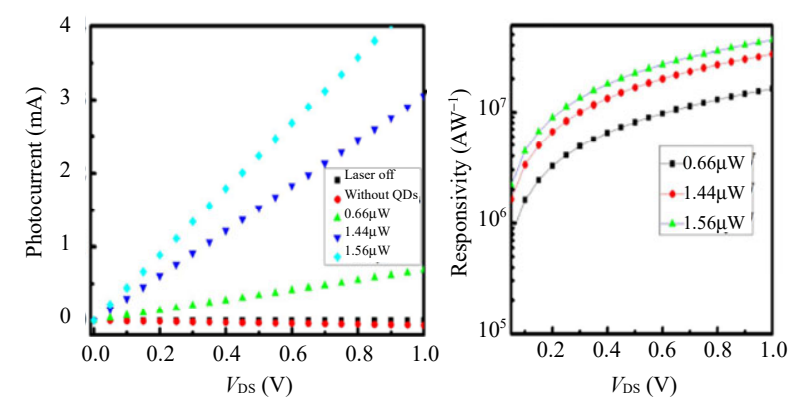

(c) photodetector composed of graphene nano ribbon (GNR) arrays integrated with the fullerene $\left(\mathrm{C}_{60}\right)$ film. $\mathrm{C}_{60}$ is an efficient trapping material for photo induced electrons and the main role of the $\mathrm{C}_{60}$ layer in the proposed device was to trap photogenerated carriers, so that longer carrier recombination lifetimes could be achieved which consequently resulted in a higher photocurrent. The schematic illustration of the device is shown in Fig.22(a). The bandgap characteristic of the hybrid nanostructures shown in Fig. 22(b) illustrates the charge transfer doping process in the system. The electron-hole pairs are generated in the GNR due to the absorption of laser light, and the electrons transfer to the defect states of the $\mathrm{C}_{60}$ film because of the bandgap alignment. It also shows that the conduction band of patterned GNR is higher than $4.4 \mathrm{eV}$ which is the theoretical Fermi level of un-patterned graphene [227]. In Fig. 22(c), the photo response of the hybrid phototransistor is shown. The photo response of the device under global irradiation was $0.4 \mathrm{~A} / \mathrm{W}$, which shows an improvement of about one order higher than that of the bare GNR phototransistor.

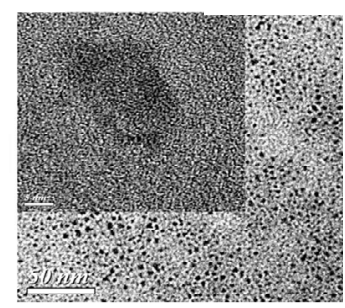

(b)

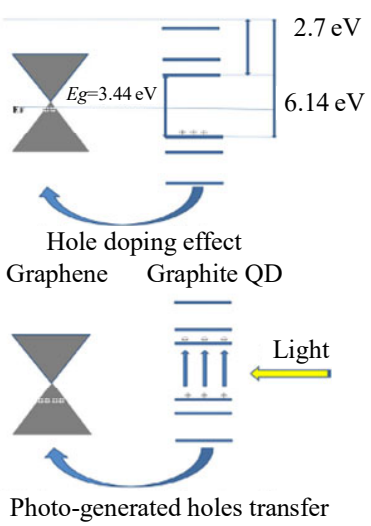

(d)

Fig. 21 Graphene decorated by quantum dots (QDs) [218]: (a) schematic diagram of all carbon-based photodetector, (b) high resolution transmission electron microscope image of graphite quantum dots, (c) photocurrent for different optical powers as a function of drain-source voltage $\left(V_{\mathrm{DS}}\right)$ and responsivity of photodetector as functions of drain-source voltage characterized under different light irradiance, and (d) schematic band diagram of graphite quantum dot-graphene hybrid device before light illumination. 


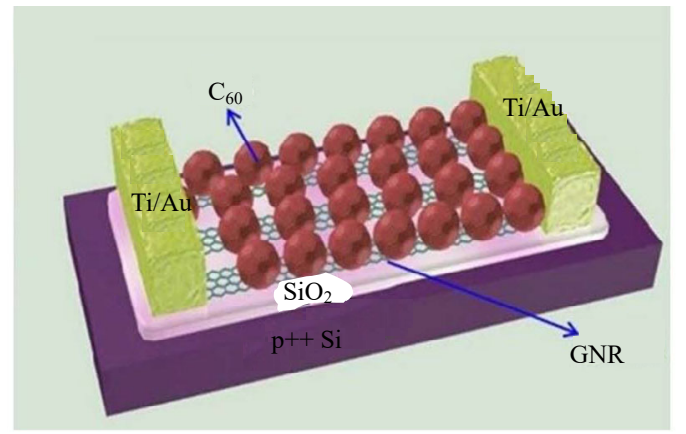

(a)

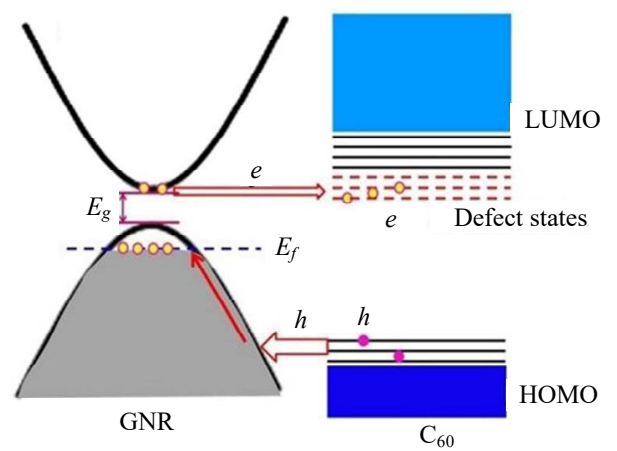

(b)

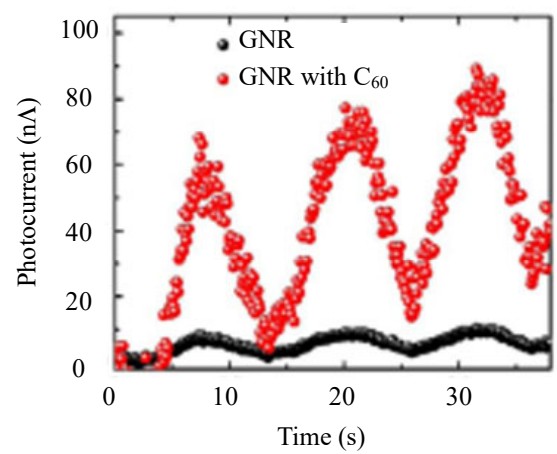

(c)

Fig. 22 Graphene nano ribbon (GNR) arrays integrated with fullerene $\left(\mathrm{C}_{60}\right)$ film photodetector [224]: (a) schematic presentation of the hybrid GNR-C $\mathrm{C}_{60}$ nanostructure, (b) the bandgap structure of GNR-C 60 hybrid nanostructures (after the photo-excitation process, the generated electrons will be trapped), and (c) the photoresponse of the hybrid phototransistor.

It is interesting to mention that in the mid-infrared region $\mathrm{C}_{60}$ had no photoresponse due to its band gap of $\approx 1.7 \mathrm{eV}$ and its main role was to introduce the trapping states which resulted in a high photocurrent as a consequence of improved trapping efficiency for the generated photoexcited electron-hole pairs. The overall mechanism of photodetection can be explained by considering the band gap of GNR $(100 \mathrm{meV})$ which is suitable enough to separate the electron-hole pairs generated by the mid-infrared light in the GNR. On the other hand, the efficient energy offset between the conduction band of the GNR and the lowest unoccupied molecular orbital (LUMO) of $\mathrm{C}_{60}$ forces the carriers to drift and trap on the $\mathrm{C}_{60}$ surface states. Therefore, more holes were circulated by the internal electrical field and more electrons were trapped in the $\mathrm{C}_{60}$ film. Although the rising and falling times of carriers were about $4 \mathrm{~s}$, due to the long trapping lifetime of the carriers in the defect and edge states of the GNR, the response of the device was improved compared with the pure GNR phototransistor and the randomly distributed graphene quantum dot photodetector [228, 229]. In the latest effort toward improvement of FGPDs, Du et al. [230] used the modulation of the dihedral angles of $\mathrm{sp}^{3}$ sites and the size/shape and fraction of $\mathrm{sp}^{3} / \mathrm{sp}^{2}$ domains to fabricate a tunable photoresponse for working as a wide-broadband photodetector. The proposed device was based on Vander Waals hetero-structures of graphene and its fluorine-functionalized derivative. They have taken the advantages of fluorine for altering the pristine graphene from a conductive semimetal to insulator (bandgap from $0 \mathrm{eV}$ to $4.1 \mathrm{eV}$ ) [231]. They have shown that, based on the degree of fluorination, some of the carbons could re-hybridize to $\mathrm{sp}^{3}$ and bonded with fluorine, while the remaining carbon was still $\mathrm{sp}^{2}$. Therefore, the variable $\mathrm{sp}^{3} / \mathrm{sp}^{2}$ fraction in fluoro-graphene and its atomic and electronic feature offered novel functionalities for photodetectors. The device has been tested in the wavelength range from $255 \mathrm{~nm}$ to $4.3 \mu \mathrm{m}$. The measured responsivity showed an improvement of more than three orders of magnitude compared with that of the graphene/graphene photodetectors.

\section{Conclusions and perspective}

FGPDs have been considered as the most 
promising optoelectronic devices for photo-sensing applications due to the simplicity of fabrication process and the unique electrical, optical, structural, and electrochemical properties of graphene. In this regards, we highlighted the most advances in fabrication of full graphene devices for photodetection applications. We have attempted to clarify feasible approaches which will most likely lead to the fabrication of more advanced FGPDs in the future. The first challenge in starting the photodetector fabrication process is to obtain a high quality graphene, therefore at the first section, a comprehensive overview on various techniques of graphene preparation, ranging from single layer to multi-layers, has been reviewed. For each technique, the pros and cons have been clarified and it is concluded that based on the specific application, one can choose an appropriate technique from the variety of approaches. For using grown graphene in various applications, transferring graphene is an essential step. Therefore, the improvements in transfer techniques from different substrates which began with applying polymer and etchants, and were improved by reducing the effect of polymer and etchant from the transfer process (polymer and etchant free) have been analyzed. Finally, transfer free techniques which can be used in special cases have been mentioned. The main challenge in each method is transferring graphene with low cracks and defects, simplifying the transfer process to reduce the cost and transferring large area graphene for industrial applications. With all advances in transfer techniques, still using polymer support and etchant is the most widely used technique for transferring graphene, particularly in full graphene photodetector fabrication. Before final step of photodetector fabrication, it is necessary to improve electronic, carrier mobility, and thermal conductivity of the graphene. This can be done by intercalation of graphene by various atoms. Accordingly, the main intercalation mechanisms are summarized and two zone evaporation techniques with the most advances in this approach are comprehensively reviewed. In the second part, before we focus on the full graphene photodetector devices, we carefully looked at the various classes of graphene photodetectors and the pros and cons on each class have been highlighted. Then, the innovative modifications which have been applied in their designs in the past few years to improve the performance of devices have been highlighted. In order to understand the basics of operation, the main parameters of the proposed device and the fundamental detection mechanisms in each device have also been discussed. Then, the FGPDs and the most recent advances for improving their performance have been summarized. The concept of FGPDs is still immature and more researches need to be done to obtain an ideal design with the acceptable performance. However, due to the simplicity of the fabrication and physics behind these devices, it is encouraging to envisage FGPDs devices to hit the market in the next years. This review can be used by the researchers as a reference to obtain the basic tool to design and fabricate more novel FGPDs with an ideal performance for the future market.

\section{Acknowledgment}

This work was supported by the Iranian National Elites Foundation (Grant No. 130/2-925) and Iran National Science Foundation (Grant No. 96008756).

Open Access This article is distributed under the terms of the Creative Commons Attribution 4.0 International License (http://creativecommons.org/licenses/by/4.0/), which permits unrestricted use, distribution, and reproduction in any medium, provided you give appropriate credit to the original author(s) and the source, provide a link to the Creative Commons license, and indicate if changes were made. 


\section{References}

[1] C. R. Dean, A. F. Young, I. Meric, C. Lee, L. Wang, S. Sorgenfrei, et al., "Boron nitride substrates for high-quality graphene electronics," Nature Nanotechnology, 2010, 5(10): 722-726.

[2] H. B. Heersche, P. Jarillo-Herrero, J. B. Oostinga, L. M. Vandersypen, and A. F. Morpurgo, "Bipolar supercurrent in graphene," Nature, 2007, 446(7131): 56-59.

[3] Z. Liu, R. Z. Wang, L. M. Liu, W. M. Lau, and H. Yan, "Bipolar doping of double-layer graphene vertical heterostructures with hydrogenated boron nitride," Physical Chemistry Chemical Physics, 2015, 17(17): 11692-11699.

[4] S. Malik, A. Vijayaraghavan, R. Erni, K. Ariga, I. Khalakhan, and J. P. Hill, "High purity graphenes prepared by a chemical intercalation method," Nanoscale, 2010, 2(10): 2139-2143.

[5] R. Murali, Y. Yang, K. Brenner, T. Beck, and J. D. Meindl, "Breakdown current density of graphene nanoribbons," Applied Physics Letters, 2009, 94(24): 243114.

[6] T. Hasan, Z. Sun, F. Wang, F. Bonaccorso, P. H. Tan, A. G. Rozhin, et al., "Nanotube-polymer composites for ultrafast photonics," Advanced Materials, 2009, 21(38-39): 3874-3899.

[7] Z. Sun, T. Hasan, F. Torrisi, D. Popa, G. Privitera, F. Wang, et al., "Graphene mode-locked ultrafast laser," ACS Nano, 2010, 4(2): 803-810.

[8] J. Wang, M. Liang, Y. Fang, T. Qiu, J. Zhang, and L. Zhi, "Rod-coating: towards large-area fabrication of uniform reduced graphene oxide films for flexible touch screens," Advanced Materials, 2012, 24(21): 2874-2878.

[9] D. Akinwande, N. Petrone, and J. Hone, "Two-dimensional flexible nanoelectronics," Nature Communications, 2014, 5(1): 1-12.

[10] T. H. Han, Y. Lee, M. R. Choi, S. H. Woo, S. H. Bae, B. H. Hong, et al., "Extremely efficient flexible organic light-emitting diodes with modified graphene anode," Nature Photonics, 2012, 6(2): 105-110.

[11] Y. Wu, X. Zou, M. Sun, Z. Cao, X. Wang, S. Huo, et al., "200 GHz maximum oscillation frequency in CVD graphene radio frequency transistors," ACS Applied Materials \& Interfaces, 2016, 8(39): 25645-25649.

[12] T. R. Nayak, H. Andersen, V. S. Makam, C. Khaw, $\mathrm{S}$. Bae, X. Xu, et al., "Graphene for controlled and accelerated osteogenic differentiation of human mesenchymal stem cells," ACS Nano, 2011, 5(6): 4670-4678.

[13]M. Liu, X. Yin, E. Ulin-Avila, B. Geng, T. Zentgraf, L. Ju, et al., "A graphene-based broadband optical modulator," Nature, 2011, 474(7349): 64-67.

[14] G. Ramakrishnan, R. Chakkittakandy, and P. C.
Planken, "Terahertz generation from graphite," Optics Express, 2009, 17(18): 16092-16099.

[15] M. J. Allen, V. C. Tung, and R. B. Kaner, "Honeycomb carbon: a review of graphene," Chemical Reviews, 2010, 110(1): 132-145.

[16] X. Miao, S. Tongay, M. K. Petterson, K. Berke, A. G. Rinzler, B. R. Appleton, et al., "High efficiency graphene solar cells by chemical doping," Nano Letters, 2012, 12(6): 2745-2750.

[17] L. Pauling, "The nature of the chemical bond-1992," Journal of Chemical Education, 1992, 69(7): 519.

[18] W. Andreoni, "The physics of fullerene-based and fullerene-related materials," Germany: Springer Science \& Business Media, 2000.

[19] R. Saito, G. Dresselhaus, and M. S. Dresselhaus, "Physical properties of carbon nanotubes," Singapore: World Scientific, 1998.

[20] J.-C. Charlier, X. Blase, and S. Roche, "Electronic and transport properties of nanotubes," Reviews of Modern Physics, 2007, 79(2): 677.

[21] H. Petroski, "The pencil: A history of design and circumstance," America: Alfred a Knopf Incorporated, 1992.

[22] K. S. Novoselov, A. K. Geim, S. V. Morozov, D. Jiang, Y. Zhang, S. V. Dubonos, et al., "Electric field effect in atomically thin carbon films," Science, 2004, 306(5696): 666-669.

[23] E. Hwang, S. Adam, and S. D. Sarma, "Carrier transport in two-dimensional graphene layers," Physical Review Letters, 2007, 98(18): 186806.

[24] F. Bonaccorso, Z. Sun, T. Hasan, and A. Ferrari, "Graphene photonics and optoelectronics," Nature Photonics, 2010, 4(9): 611-622.

[25] A. C. Neto, F. Guinea, N. M. Peres, K. S. Novoselov, and A. K. Geim, "The electronic properties of graphene," Reviews of Modern Physics, 2009, 81(1): 109-162.

[26] P. A. Lee and T. Ramakrishnan, "Disordered electronic systems," Reviews of Modern Physics, 1985, 57(2): 287.

[27] K. S. Novoselov, A. K. Geim, S. Morozov, D. Jiang, M. Katsnelson, I. Grigorieva, et al., "Two-dimensional gas of massless Dirac fermions in graphene," Nature, 2005, 438(7065): 197-200.

[28] C. Itzykson and J. B. Zuber, "Quantum field theory," England: Courier Corporation, 2006.

[29]Z. S. Wu, A. Winter, L. Chen, Y. Sun, A. Turchanin, $\mathrm{X}$. Feng, et al., "Three-dimensional nitrogen and boron co-doped graphene for high-performance all-solid-state supercapacitors," Advanced Materials, 2012, 24(37): 5130-5135.

[30] M. S. Dresselhaus and G. Dresselhaus, "Intercalation compounds of graphite," Advances in Physics, 2002, 51(1): 1-186.

[31] O. Leenaerts, B. Partoens, and F. Peeters, "Adsorption of $\mathrm{H}_{2} \mathrm{O}, \mathrm{NH}_{3}, \mathrm{CO}, \mathrm{NO}_{2}$, and $\mathrm{NO}$ on 
graphene: a first-principles study," Physical Review B, 2008, 77(12): 125416.

[32] T. Wehling, K. Novoselov, S. Morozov, E. Vdovin, M. Katsnelson, A. Geim, et al., "Molecular doping of graphene," Nano Letters, 2008, 8(1): 173-177.

[33] A. S. Mayorov, R. V. Gorbachev, S. V. Morozov, L. Britnell, R. Jalil, L. A. Ponomarenko, et al., "Micrometer-scale ballistic transport in encapsulated graphene at room temperature," Nano Letters, 2011, 11(6): 2396-2399.

[34] J. Baringhaus, M. Ruan, F. Edler, A. Tejeda, M. Sicot, A. T. Ibrahimi, et al., "Exceptional ballistic transport in epitaxial graphene nanoribbons," Nature, 2014, 506(7488): 349-354.

[35 C. Lee, X. Wei, J. W. Kysar, and J. Hone, "Measurement of the elastic properties and intrinsic strength of monolayer graphene," Science, 2008, 321(5887): 385-388.

[36] F. Liu, P. Ming, and J. Li, “Ab initio calculation of ideal strength and phonon instability of graphene under tension," Physical Review B, 2007, 76(6): 064120

[37] A. A. Balandin, "Thermal properties of graphene and nanostructured carbon materials," Nature Materials, 2011, 10(8): 569-581.

[38] J. S. Bunch, S. S. Verbridge, J. S. Alden, A. M. Van Der Zande, J. M. Parpia, H. G. Craighead, et al., "Impermeable atomic membranes from graphene sheets," Nano Letters, 2008, 8(8): 2458-2462.

[39] R. R. Nair, P. Blake, A. N. Grigorenko, K. S. Novoselov, T. J. Booth, T. Stauber, et al., "Fine structure constant defines visual transparency of graphene," Science, 2008, 320(5881): 1308.

[40] J. Moser, A. Barreiro, and A. Bachtold, "Current-induced cleaning of graphene," Applied Physics Letters, 2007, 91(16): 163513.

[41] Y. Zhang, Y. W. Tan, H. L. Stormer, and P. Kim, "Experimental observation of the quantum Hall effect and Berry's phase in graphene," Nature, 2005, 438(7065): 201-204.

[42] X. Du, I. Skachko, F. Duerr, A. Luican, and E. Y. Andrei, "Fractional quantum Hall effect and insulating phase of Dirac electrons in graphene," Nature, 2009, 462(7270): 192-195.

[43] A. K. Geim and K. S. Novoselov, "The rise of graphene," Nature Materials, 2007, 6: 183-191.

[44] M. Mucha-Kruczyński, O. Tsyplyatyev, A. Grishin, E. McCann, V. I. Fal'ko, A. Bostwick, et al., "Characterization of graphene through anisotropy of constant-energy maps in angle-resolved photoemission," Physical Review B, 2008, 77(19): 195403.

[45] C. Casiraghi, A. Hartschuh, E. Lidorikis, H. Qian, H. Harutyunyan, T. Gokus, et al., "Rayleigh imaging of graphene and graphene layers," Nano Letters, 2007, 7(9): 2711-2717.

[46] R. A. Schultz, M. C. Jensen, and R. C. Bradt,
"Single crystal cleavage of brittle materials," International Journal of Fracture, 1994, 65(4): 291-312.

[47] K. Novoselov, A. K. Geim, S. Morozov, D. Jiang, M. Katsnelson, I. Grigorieva, et al., "Two-dimensional gas of massless Dirac fermions in graphene," Nature, 2005, 438(7065): 197-200.

[48] G. Wallis and D. I. Pomerantz, "Field assisted glass-metal sealing," Journal of Applied Physics, 1969, 40(10): 3946-3949.

[49] E. Collart, A. Shukla, F. Gélébart, M. Morand, C. Malgrange, N. Bardou, et al., "Spherically bent analyzers for resonant inelastic X-ray scattering with intrinsic resolution below $200 \mathrm{meV}$," Journal of Synchrotron Radiation, 2005, 12(4): 473-478.

[50] J. N. Israelachvili, "Intermolecular and surface forces: revised third edition," America: Academic Press, 2011.

[51] Y. Hernandez, V. Nicolosi, M. Lotya, F. M. Blighe, Z. Sun, S. De, et al., "High-yield production of graphene by liquid-phase exfoliation of graphite," Nature Nanotechnology, 2008, 3(9): 563-568.

[52] F. Bonaccorso, A. Lombardo, T. Hasan, Z. Sun, L. Colombo, and A. C. Ferrari, "Production and processing of graphene and $2 \mathrm{~d}$ crystals," Materials Today, 2012, 15(12): 564-589.

[53] A. O’Neill, U. Khan, P. N. Nirmalraj, J. Boland, and J. N. Coleman, "Graphene dispersion and exfoliation in low boiling point solvents," The Journal of Physical Chemistry C, 2011, 115(13): 5422-5428.

[54] M. Lotya, P. J. King, U. Khan, S. De, and J. N. Coleman, "High-concentration, surfactantstabilized graphene dispersions," ACS Nano, 2010, 4(6): 3155-3162.

[55] O. M. Maragó, F. Bonaccorso, R. Saija, G. Privitera, P. G. Gucciardi, M. A. Iatì, et al., "Brownian motion of graphene," ACS Nano, 2010, 4(12): 7515-7523.

[56] T. Hasan, F. Torrisi, Z. Sun, D. Popa, V. Nicolosi, G. Privitera, et al., "Solution-phase exfoliation of graphite for ultrafast photonics," Physica Status Solidi (b), 2010, 247(11-12): 2953-2957.

[57] U. Khan, A. O'Neill, M. Lotya, S. De, and J. N. Coleman, "High-concentration solvent exfoliation of graphene," Small, 2010, 6(7): 864-871.

[58] J. Hassoun, F. Bonaccorso, M. Agostini, M. Angelucci, M. G. Betti, R. Cingolani, et al., "An advanced lithium-ion battery based on a graphene anode and a lithium iron phosphate cathode," Nano Letters, 2014, 14(8): 4901-4906.

[59] T. Mason and D. Peters, "An introduction to the uses of power ultrasound in chemistry. in Practical Sonochemistry (Second Edition)," Sawston Cambridge: Woodhead Publishing, 2002, pp. 1-48.

[60] L. Huang, Y. Huang, J. Liang, X. Wan, and Y. Chen, "Graphene-based conducting inks for direct 
inkjet printing of flexible conductive patterns and their applications in electric circuits and chemical sensors," Nano Research, 2011, 4(7): 675-684.

[61] F. Torrisi, T. Hasan, W. Wu, Z. Sun, A. Lombardo, T. S. Kulmala, et al., "Inkjet-printed graphene electronics," ACS Nano, 2012, 6(4): 2992-3006.

[62] J. N. Coleman, M. Lotya, A. O’Neill, S. D. Bergin, P. J. King, U. Khan, et al., "Two-dimensional nanosheets produced by liquid exfoliation of layered materials," Science, 2011, 331(6017): 568-571.

[63] M. Lotya, Y. Hernandez, P. J. King, R. J. Smith, V. Nicolosi, L. S. Karlsson, et al., "Liquid phase production of graphene by exfoliation of graphite in surfactant/water solutions," Journal of the American Chemical Society, 2009, 131(10): 3611-3620.

[64] H. An, W. J. Lee, and J. Jung, "Graphene synthesis on Fe foil using thermal CVD," Current Applied Physics, 2011, 11(4): S81-S85.

[65] Y. Xue, B. Wu, Y. Guo, L. Huang, L. Jiang, J. Chen, et al., "Synthesis of large-area, few-layer graphene on iron foil by chemical vapor deposition," Nano Research, 2011, 4(12): 1208-1214.

[66]M. E. Ramón, A. Gupta, C. Corbet, D. A. Ferrer, H. C. Movva, G. Carpenter, et al., "CMOS-compatible synthesis of large-area, high-mobility graphene by chemical vapor deposition of acetylene on cobalt," ACS nano, 2011, 5(9): 7198-7204.

[67]H. Ago, Y. Ito, N. Mizuta, K. Yoshida, B. Hu, C. M. Orofeo, et al., "Epitaxial chemical vapor deposition growth of single-layer graphene over cobalt film crystallized on sapphire," ACS Nano, 2010, 4(12): 7407-7414.

[68] A. Reina, X. Jia, J. Ho, D. Nezich, H. Son, V. Bulovic, et al., "Large area, few-layer graphene films on arbitrary substrates by chemical vapor deposition," Nano Letters, 2008, 9(1): 30-35.

[69] Z. Li, H. Zhu, D. Xie, K. Wang, A. Cao, J. Wei, et al., "Flame synthesis of few-layered graphene/graphite films," Chemical Communications, 2011, 47(12): 3520-3522.

[70] A. Guermoune, T. Chari, F. Popescu, S. S. Sabri, J. Guillemette, H. S. Skulason, et al., "Chemical vapor deposition synthesis of graphene on copper with methanol, ethanol, and propanol precursors," Carbon, 2011, 49(13): 4204-4210.

[71] Y. Wang, Y. Zheng, X. Xu, E. Dubuisson, Q. Bao, J. $\mathrm{Lu}$, et al., "Electrochemical delamination of CVD-grown graphene film: toward the recyclable use of copper catalyst," ACS Nano, 2011, 5(12): 9927-9933.

[72] X. Chen, L. Zhang, and S. Chen, "Large area CVD growth of graphene," Synthetic Metals, 2015, 210: 95-108.

[73] S. Stankovich, D. A. Dikin, G. H. Dommett, K. M.
Kohlhaas, E. J. Zimney, E. A. Stach, et al., "Graphene-based composite materials," Nature, 2006, 442(7100): 282-286.

[74] C. Mattevi, H. Kim, and M. Chhowalla, "A review of chemical vapour deposition of graphene on copper," Journal of Materials Chemistry, 2011, 21(10): 3324-3334.

[75]S. Bae, H. Kim, Y. Lee, X. Xu, J. S. Park, Y. Zheng, et al., "Roll-to-roll production of 30-inch graphene films for transparent electrodes," Nature Nanotechnology, 2010, 5(8): 574-578.

[76] A. Malesevic, R. Vitchev, K. Schouteden, A. Volodin, L. Zhang, G. Van Tendeloo, et al., "Synthesis of few-layer graphene via microwave plasma-enhanced chemical vapour deposition," Nanotechnology, 2008, 19(30): 305604.

[77] J. Wang, M. Zhu, R. A. Outlaw, X. Zhao, D. M. Manos, and B. C. Holloway, "Synthesis of carbon nanosheets by inductively coupled radio-frequency plasma enhanced chemical vapor deposition," Carbon, 2004, 42(14): 2867-2872.

[78] V. Krivchenko, V. Dvorkin, N. Dzbanovsky, M. Timofeyev, A. Stepanov, A. Rakhimov, et al., "Evolution of carbon film structure during its catalyst-free growth in the plasma of direct current glow discharge," Carbon, 2012, 50(4): 1477-1487.

[79] J. Chan, A. Venugopal, A. Pirkle, S. McDonnell, D. Hinojos, C. W. Magnuson, et al., "Reducing extrinsic performance-limiting factors in graphene grown by chemical vapor deposition," ACS Nano, 2012, 6(4): 3224-3229.

[80] N. Petrone, C. R. Dean, I. Meric, A. M. van Der Zande, P. Y. Huang, L. Wang, et al., "Chemical vapor deposition-derived graphene with electrical performance of exfoliated graphene," Nano Letters, 2012, 12(6): 2751-2756.

[81] J. H. Deng, R. T. Zheng, Y. Zhao, and G. A. Cheng, "Vapor-solid growth of few-layer graphene using radio frequency sputtering deposition and its application on field emission," ACS Nano, 2012, 6(5): 3727-3733.

[82] M. I. Ionescu, X. Sun, and B. Luan, "Multilayer graphene synthesized using magnetron sputtering for planar supercapacitor application," Canadian Journal of Chemistry, 2014, 93(2): 160-164.

[83] D. T. Oldfield, D. G. McCulloch, C. P. Huynh, K. Sears, and S. C. Hawkins, "Multilayered graphene films prepared at moderate temperatures using energetic physical vapour deposition," Carbon, 2015, 94: 378-385.

[84] G. Pan, B. Li, M. Heath, D. Horsell, M. L. Wears, L. Al Taan, et al., "Transfer-free growth of graphene on $\mathrm{SiO}_{2}$ insulator substrate from sputtered carbon and nickel films," Carbon, 2013, 65: 349-358.

[85] X. Dong, S. Liu, H. Song, and P. Gu, "Growth of large-area, few-layer graphene by femtosecond 
pulsed laser deposition with double-layer $\mathrm{Ni}$ catalyst," Journal of Materials Science, 2017, 52(4): 2060-2065.

[86] R. K. Vijayaraghavan, C. Gaman, B. Jose, A. P. McCoy, T. Cafolla, P. J. McNally, et al., "Pulsed-plasma physical vapor deposition approach toward the facile synthesis of multilayer and monolayer graphene for anticoagulation applications," ACS Applied Materials \& Interfaces, 2016, 8(7): 4878-4886.

[87]Z. S. Wu, W. Ren, L. Gao, J. Zhao, Z. Chen, B. Liu, et al., "Synthesis of graphene sheets with high electrical conductivity and good thermal stability by hydrogen arc discharge exfoliation," ACS Nano, 2009, 3(2): 411-417.

[88] Z. Ying, R. Hettich, R. Compton, and R. Haufler, "Synthesis of nitrogen-doped fullerenes by laser ablation," Journal of Physics B: Atomic, Molecular and Optical Physics, 1996, 29(21): 4935.

[89] G. Radhakrishnan, P. Adams, and L. Bernstein, "Plasma characterization and room temperature growth of carbon nanotubes and nano-onions by excimer laser ablation," Applied Surface Science, 2007, 253(19): 7651-7655.

[90] A. Modabberasl, P. Kameli, M. Ranjbar, H. Salamati, and R. Ashiri, "Fabrication of DLC thin films with improved diamond-like carbon character by the application of external magnetic field," Carbon, 2015, 94: 485-493.

[91] A. M. Asl, P. Kameli, M. Ranjbar, H. Salamati, and M. Jannesari, "Correlations between microstructure and hydrophobicity properties of pulsed laser deposited diamond-like carbon films," Superlattices and Microstructures, 2015, 81: 64-79.

[92] H. Zhang and P. X. Feng, "Fabrication and characterization of few-layer graphene," Carbon, 2010, 48(2): 359-364.

[93] F. Larki, P. Kameli, H. Nikmanesh, M. Jafari, and H. Salamati, "The influence of external magnetic field on the pulsed laser deposition growth of graphene on nickel substrate at room temperature," Diamond and Related Materials, 2019, 93: 233-240.

[94] P. J. McNally and S. Daniels, "Pulsed plasma physical vapour deposition approach towards the facile synthesis of multilayer and monolayer graphene for anticoagulation applications," $A C S$ Applied Materials \& Interfaces, 2016, 8(7): 4878-4886.

[95] E. G. Acheson, "Manufacture of graphite," in Google Patents, 1896, US568 323A.

[96] X. Liang, B. A. Sperling, I. Calizo, G. Cheng, C. A. Hacker, Q. Zhang, et al., "Toward clean and crackless transfer of graphene," ACS Nano, 2011, 5(11): 9144-9153.

[97] X. Li, Y. Zhu, W. Cai, M. Borysiak, B. Han, D.
Chen, et al., "Transfer of large-area graphene films for high-performance transparent conductive electrodes," Nano Letters, 2009, 9(12): 4359-4363.

[98] Y. Zhang, J. P. Small, W. V. Pontius, and P. Kim, "Fabrication and electric-field-dependent transport measurements of mesoscopic graphite devices," Applied Physics Letters, 2005, 86(7): 073104.

[99]S. Unarunotai, Y. Murata, C. E. Chialvo, H. S. Kim, S. MacLaren, N. Mason, et al., "Transfer of graphene layers grown on $\mathrm{SiC}$ wafers to other substrates and their integration into field effect transistors," Applied Physics Letters, 2009, 95(20): 202101.

[100]J. D. Caldwell, T. J. Anderson, J. C. Culbertson, G. G. Jernigan, K. D. Hobart, F. J. Kub, et al., "Technique for the dry transfer of epitaxial graphene onto arbitrary substrates," ACS Nano, 2010, 4(2): 1108-1114.

[101]L. Chen, Z. Kong, S. Yue, J. Liu, J. Deng, Y. Xiao, et al., "Growth of uniform monolayer graphene using iron-group metals via the formation of an antiperovskite layer," Chemistry of Materials, 2015, 27(24): 8230-8236.

[102] E. Sutter, P. Albrecht, and P. Sutter, "Graphene growth on polycrystalline Ru thin films," Applied Physics Letters, 2009, 95(13): 133109.

[103] Y. Que, W. Xiao, X. Fei, H. Chen, L. Huang, S. $\mathrm{Du}$, et al., "Epitaxial growth of large-area bilayer graphene on Ru (0001)," Applied Physics Letters, 2014, 104(9): 093110.

[104] S. Wang, Y. Pei, X. Wang, H. Wang, Q. Meng, H. Tian, et al., "Synthesis of graphene on a polycrystalline Co film by radio-frequency plasma-enhanced chemical vapour deposition," Journal of Physics D: Applied Physics, 2010, 43(45): 455402.

[105] J. Coraux, A. T. N'Diaye, C. Busse, and T. Michely, "Structural coherency of graphene on Ir (111)," Nano Letters, 2008, 8(2): 565-570.

[106] C. Vo-Van, A. Kimouche, A. Reserbat-Plantey, O. Fruchart, P. Bayle-Guillemaud, N. Bendiab, et al., "Epitaxial graphene prepared by chemical vapor deposition on single crystal thin iridium films on sapphire," Applied Physics Letters, 2011, 98(18): 181903.

[107] B. J. Kang, J. H. Mun, C. Y. Hwang, and B. J. Cho, "Monolayer graphene growth on sputtered thin film platinum," Journal of Applied Physics, 2009, 106(10): 104309.

[108] T. Oznuluer, E. Pince, E. O. Polat, O. Balci, O. Salihoglu, and C. Kocabas, "Synthesis of graphene on gold," Applied Physics Letters, 2011, 98(18): 183101.

[109]K. S. Kim, Y. Zhao, H. Jang, S. Y. Lee, J. M. Kim, K. S. Kim, et al., "Large-scale pattern growth of graphene films for stretchable transparent 
electrodes," Nature, 2009, 457(7230): 706-710.

[110] Y. Lee, S. Bae, H. Jang, S. Jang, S. E. Zhu, S. H. Sim, et al., "Wafer-scale synthesis and transfer of graphene films," Nano Letters, 2010, 10(2): 490-493.

[111] L. Jiao, B. Fan, X. Xian, Z. Wu, J. Zhang, and Z. Liu, "Creation of nanostructures with poly (methyl methacrylate)-mediated nanotransfer printing," Journal of the American Chemical Society, 2008, 130(38): 12612-12613.

[112] Y. C. Lin, C. Jin, J. C. Lee, S. F. Jen, K. Suenaga, and P. W. Chiu, "Clean transfer of graphene for isolation and suspension," ACS Nano, 2011, 5(3): 2362-2368.

[113] J. Song, F. Y. Kam, R. Q. Png, W. L. Seah, J. M. Zhuo, G. K. Lim, et al., "A general method for transferring graphene onto soft surfaces," Nature Nanotechnology, 2013, 8(5): 356-362.

[114] J. D. Wood, G. P. Doidge, E. A. Carrion, J. C. Koepke, J. A. Kaitz, I. Datye, et al., "Annealing free, clean graphene transfer using alternative polymer scaffolds," Nanotechnology, 2015, 26(5): 055302.

[115] T. Matsumae, A. D. Koehler, T. Suga, and K. D. Hobart, "A scalable clean graphene transfer process using polymethylglutarimide as a support scaffold," Journal of the Electrochemical Society, 2016, 163(6): E159-E161.

[116] A. Nath, A. D. Koehler, G. G. Jernigan, V. D. Wheeler, J. K. Hite, S. C. Hernández, et al., "Achieving clean epitaxial graphene surfaces suitable for device applications by improved lithographic process," Applied Physics Letters, 2014, 104(22): 224102.

[117] J. Lee, Y. Kim, H. J. Shin, C. Lee, D. Lee, S. Lee, et al., "Crack-release transfer method of wafer-scale grown graphene onto large-area substrates," ACS Applied Materials \& Interfaces, 2014, 6(15): 12588-12593.

[118] H. Park, P. R. Brown, V. Bulović, and J. Kong, "Graphene as transparent conducting electrodes in organic photovoltaics: studies in graphene morphology, hole transporting layers, and counter electrodes," Nano Letters, 2011, 12(1): 133-140.

[119] Y. Han, L. Zhang, X. Zhang, K. Ruan, L. Cui, Y. Wang, et al., "Clean surface transfer of graphene films via an effective sandwich method for organic light-emitting diode applications," Journal of Materials Chemistry C, 2014, 2(1): 201-207.

[120]H. J. Jeong, H. Y. Kim, S. Y. Jeong, J. T. Han, K. J. Baeg, J. Y. Hwang, et al., "Improved transfer of chemical-vapor-deposited graphene through modification of intermolecular interactions and solubility of poly (methylmethacrylate) layers," Carbon, 2014, 66: 612-618.

[121]W. Regan, N. Alem, B. Alemán, B. Geng, Ç. Girit, L. Maserati, et al., "A direct transfer of layer-area graphene," Applied Physics Letters, 2010, 96(11): 113102.

[122] S. Tanabe, K. Furukawa, and H. Hibino, "Etchant-free and damageless transfer of monolayer and bilayer graphene grown on $\mathrm{SiC}$," Japanese Journal of Applied Physics, 2014, 53(11): 115101.

[123] M. P. Levendorf, C. S. Ruiz-Vargas, S. Garg, and J. Park, "Transfer-free batch fabrication of single layer graphene transistors," Nano Letters, 2009, 9(12): 4479-4483.

[124] A. Ismach, C. Druzgalski, S. Penwell, A. Schwartzberg, M. Zheng, A. Javey, et al., "Direct chemical vapor deposition of graphene on dielectric surfaces," Nano Letters, 2010, 10(5): 1542-1548.

[125] S. J. Byun, H. Lim, G. Y. Shin, T. H. Han, S. H. Oh, J.-H. Ahn, et al., "Graphenes converted from polymers," The Journal of Physical Chemistry Letters, 2011, 2(5): 493-497.

[126] H. J. Shin, W. M. Choi, S. M. Yoon, G. H. Han, Y. S. Woo, E. S. Kim, et al., "Transfer-free growth of few-layer graphene by self-assembled monolayers," Advanced Materials, 2011, 23(38): 4392-4397.

[127] C. Y. Su, A. Y. Lu, C. Y. Wu, Y. T. Li, K. K. Liu, W. Zhang, et al., "Direct formation of wafer scale graphene thin layers on insulating substrates by chemical vapor deposition," Nano Letters, 2011, 11(9): 3612-3616.

[128] K. Fujita, K. Banno, H. Aryal, and T. Egawa, "Graphene layer growth on silicon substrates with nickel film by pulse arc plasma deposition," Applied Physics Letters, 2012, 101(16): 163109.

[129] C. Mattevi, F. Colléaux, H. Kim, Y. H. Lin, K. T. Park, M. Chhowalla, et al., "Solution-processable organic dielectrics for graphene electronics," Nanotechnology, 2012, 23(34): 344017.

[130] K. Banno, M. Mizuno, K. Fujita, T. Kubo, M. Miyoshi, T. Egawa, et al., "Transfer-free graphene synthesis on insulating substrates via agglomeration phenomena of catalytic nickel films," Applied Physics Letters, 2013, 103(8): 082112.

[131] M. Miyoshi, M. Mizuno, K. Banno, T. Kubo, T. Egawa, and T. Soga, "Study on transfer-free graphene synthesis process utilizing spontaneous agglomeration of catalytic $\mathrm{Ni}$ and Co metals," Materials Research Express, 2015, 2(1): 015602.

[132] S. M. Sze and K. K. Ng, "Physics of semiconductor devices," New Jersey: John Wiley \& Sons, 2006.

[133] A. C. Ferrari, F. Bonaccorso, V. Fal'Ko, K. S. Novoselov, S. Roche, P. Bøggild, et al., "Science and technology roadmap for graphene, related two-dimensional crystals, and hybrid systems," Nanoscale, 2015, 7(11): 4598-4810. 
[134] S. C. Dhanabalan, J. S. Ponraj, H. Zhang, and Q. Bao, "Present perspectives of broadband photodetectors based on nanobelts, nanoribbons, nanosheets and the emerging 2D materials," Nanoscale, 2016, 8(12): 6410-6434.

[135] K. Zhang, T. Zhang, G. Cheng, T. Li, S. Wang, W. Wei, et al., "Interlayer transition and infrared photodetection in atomically thin type-II $\mathrm{MoTe}_{2} / \mathrm{MoS}_{2}$ van der Waals heterostructures," $A C S$ Nano, 2016, 10(3): 3852-3858.

[136] K. F. Mak and J. Shan, "Photonics and optoelectronics of 2D semiconductor transition metal dichalcogenides," Nature Photonics, 2016, 10(4): 216-226.

[137] X. Congxin and L. Jingbo, "Recent advances in optoelectronic properties and applications of two-dimensional metal chalcogenides," Journal of Semiconductors, 2016, 37(5): 051001.

[138] K. Novoselov and A. C. Neto, "Two-dimensional crystals-based heterostructures: materials with tailored properties," Physica Scripta, 2012, 2012(T146): 014006.

[139] P. Hu, Z. Wen, L. Wang, P. Tan, and K. Xiao, "Synthesis of few-layer GaSe nanosheets for high performance photodetectors," ACS Nano, 2012, 6(7): 5988-5994.

[140] M. Houssa, A. Dimoulas, and A. Molle, " $2 D$ materials for nanoelectronics," Florida: CRC Press, 2016.

[141] X. Chen, X. Sun, J. Jiang, Q. Liang, Q. Yang, and R. Meng, "Electrical and optical properties of germanene on single-layer $\mathrm{BeO}$ substrate," The Journal of Physical Chemistry C, 2016, 120(36): 20350-20356.

[142] R. Peale, M. Ishigami, and C. W. Smith, "Plasmonic phototransistor," in Google Patents, 2016, US20150109606A1.

[143 Y. Guo, C. Liu, H. Tanaka, and E. Nakamura, "Air-stable and solution-processable perovskite photodetectors for solar-blind UV and visible light," The Journal of Physical Chemistry Letters, 2015, 6(3): 535-539.

[144] L. Dou, Y. M. Yang, J. You, Z. Hong, W. H. Chang, G. Li, et al., "Solution-processed hybrid perovskite photodetectors with high detectivity," Nature Communications, 2014, 5(1): 1-6.

[145] J. M. Dawlaty, S. Shivaraman, M. Chandrashekhar, F. Rana, and M. G. Spencer, "Measurement of ultrafast carrier dynamics in epitaxial graphene," Applied Physics Letters, 2008, 92(4): 042116.

[146] D. Brida, A. Tomadin, C. Manzoni, Y. J. Kim, A. Lombardo, S. Milana, et al., "Ultrafast collinear scattering and carrier multiplication in graphene," Nature Communications, 2013, 4(1): 1-9.

[147] E. J. Lee, K. Balasubramanian, R. T. Weitz, M. Burghard, and K. Kern, "Contact and edge effects in graphene devices," Nature Nanotechnology, 2008, 3(8): 486-490.

[148] F. Xia, T. Mueller, R. Golizadeh-Mojarad, M. Freitag, Y. M. Lin, J. Tsang, et al., "Photocurrent imaging and efficient photon detection in a graphene transistor," Nano Letters, 2009, 9(3): 1039-1044.

[149] F. Koppens, T. Mueller, P. Avouris, A. Ferrari, M. Vitiello, and M. Polini, "Photodetectors based on graphene, other two-dimensional materials and hybrid systems," Nature Nanotechnology, 2014, 9(10): 780-793.

[150] D. B. Farmer, R. Golizadeh-Mojarad, V. Perebeinos, Y. M. Lin, G. S. Tulevski, J. C. Tsang, et al., "Chemical doping and electron-hole conduction asymmetry in graphene devices," Nano Letters, 2009, 9(1): 388-392.

[151] M. C. Lemme, F. H. Koppens, A. L. Falk, M. S. Rudner, H. Park, L. S. Levitov, et al., "Gate-activated photoresponse in a graphene $\mathrm{p}-\mathrm{n}$ junction," Nano Letters, 2011, 11(10): 4134-4137.

[152] E. C. Peters, E. J. Lee, M. Burghard, and K. Kern, "Gate dependent photocurrents at a graphene pn junction," Applied Physics Letters, 2010, 97(19): 193102.

[153] M. Freitag, T. Low, F. Xia, and P. Avouris, "Photoconductivity of biased graphene," Nature Photonics, 2013, 7(1): 53-59.

[154] T. Mueller, F. Xia, and P. Avouris, "Graphene photodetectors for high-speed optical communications," Nature Photonics, 2010, 4(5): 297-301.

[155] S. Piscanec, M. Lazzeri, F. Mauri, A. Ferrari, and J. Robertson, "Kohn anomalies and electron-phonon interactions in graphite," Physical Review Letters, 2004, 93(18): 185503.

[156] X. Xu, N. M. Gabor, J. S. Alden, A. M. van der Zande, and P. L. McEuen, "Photo-thermoelectric effect at a graphene interface junction," Nano Letters, 2010, 10(2): 562-566.

[157] N. M. Gabor, J. C. Song, Q. Ma, N. L. Nair, T. Taychatanapat, K. Watanabe, et al., "Hot carrier-assisted intrinsic photoresponse in graphene," Science, 2011, 334(6056): 648-652.

[158] M. Freitag, T. Low, and P. Avouris, "Increased responsivity of suspended graphene photodetectors," Nano Letters, 2013, 13(4): 1644-1648.

[159] P. Richards, "Bolometers for infrared and millimeter waves," Journal of Applied Physics, 1994, 76(1): 1-24.

[160] H. Maruska, M. Hicks, T. Moustakas, and R. Friedman, "Optically controlled amorphous silicon photosensitive device," IEEE Transactions on Electron Devices, 1984, 31(9): 1343-1345.

[161] J. Hou and S. Fonash, "Quantum efficiencies greater than unity: a computer study of a 
photogating effect in amorphous silicon $p-i-n$ devices," Applied Physics Letters, 1992, 61(2): 186-188.

[162] M. Dyakonov and M. Shur, "Shallow water analogy for a ballistic field effect transistor: new mechanism of plasma wave generation by dc current," Physical Review Letters, 1993, 71(15): 2465.

[163] M. Dyakonov and M. Shur, "Detection, mixing, and frequency multiplication of terahertz radiation by two-dimensional electronic fluid," IEEE Transactions on Electron Devices, 1996, 43(3): 380-387.

[164] A. Tomadin and M. Polini, "Theory of the plasma-wave photoresponse of a gated graphene sheet," Physical Review B, 2013, 88(20): 205426.

[165] A. Muraviev, S. Rumyantsev, G. Liu, A. Balandin, W. Knap, and M. Shur, "Plasmonic and bolometric terahertz detection by graphene field-effect transistor," Applied Physics Letters, 2013, 103(18): 181114.

[166] K. Y. Yeung, J. Chee, H. Yoon, Y. Song, J. Kong, and D. Ham, "Far-infrared graphene plasmonic crystals for plasmonic band engineering," Nano Letters, 2014, 14(5): 2479-2484.

[167] L. Wang, X. Chen, and W. Lu, "Intrinsic photo-conductance triggered by the plasmonic effect in graphene for terahertz detection," Nanotechnology, 2015, 27(3): 035205.

[168] T. Mueller, F. Xia, M. Freitag, J. Tsang, and P. Avouris, "Role of contacts in graphene transistors: a scanning photocurrent study," Physical Review B, 2009, 79(24): 245430.

[169] J. Park, Y. Ahn, and C. Ruiz-Vargas, "Imaging of photocurrent generation and collection in single-layer graphene," Nano Letters, 2009, 9(5): $1742-1746$.

[170] T. J. Echtermeyer, P. Nene, M. Trushin, R. V. Gorbachev, A. L. Eiden, S. Milana, et al., "Photothermoelectric and photoelectric contributions to light detection in metal-graphene-metal photodetectors," Nano Letters, 2014, 14(7): 3733-3742.

[171] X. Cai, R. J. Suess, H. D. Drew, T. E. Murphy, J. Yan, and M. S. Fuhrer, "Pulsed near-IR photoresponse in a Bi-metal contacted graphene photodetector," Scientific Reports, 2015, 5: 14803.

[172] S. Schuler, D. Schall, D. Neumaier, L. Dobusch, O. Bethge, B. Schwarz, et al., "Controlled generation of a $\mathrm{p}-\mathrm{n}$ junction in a waveguide integrated graphene photodetector," Nano Letters, 2016, 16(11): 7107-7112.

[173] X. Cai, A. B. Sushkov, R. J. Suess, M. M. Jadidi, G. S. Jenkins, L. O. Nyakiti, et al., "Sensitive room-temperature terahertz detection via the photothermoelectric effect in graphene," Nature Nanotechnology, 2014, 9(10): 814-819.
[174] D. Sun, G. Aivazian, A. M. Jones, J. S. Ross, W. Yao, D. Cobden, et al., "Ultrafast hot-carrier-dominated photocurrent in graphene," Nature Nanotechnology, 2012, 7(2): 114-118.

[175] L. Prechtel, L. Song, D. Schuh, P. Ajayan, W. Wegscheider, and A. W. Holleitner, "Time-resolved ultrafast photocurrents and terahertz generation in freely suspended graphene," Nature Communications, 2012, 3(1): 1-7.

[176]T. Mueller, M. Furchi, A. Urich, and A. Pospischil, "Metal-graphene-metal photodetectors," in SPIE LASE, California, 2013, pp. 86001H.

[177] T. Limmer, J. Feldmann, and E. Da Como, "Carrier lifetime in exfoliated few-layer graphene determined from intersubband optical transitions," Physical Review Letters, 2013, 110(21): 217406.

[178] P. A. George, J. Strait, J. Dawlaty, S. Shivaraman, M. Chandrashekhar, F. Rana, et al., "Ultrafast optical-pump terahertz-probe spectroscopy of the carrier relaxation and recombination dynamics in epitaxial graphene," Nano Letters, 2008, 8(12): 4248-4251.

[179] A. Di Bartolomeo, "Graphene Schottky diodes: an experimental review of the rectifying graphene/semiconductor heterojunction," Physics Reports, 2016, 606: 1-58.

[180] X. An, F. Liu, Y. J. Jung, and S. Kar, "Tunable graphene-silicon heterojunctions for ultrasensitive photodetection," Nano Letters, 2013, 13(3): 909-916.

[181] T. Echtermeyer, L. Britnell, P. Jasnos, A. Lombardo, R. Gorbachev, A. Grigorenko, et al., "Strong plasmonic enhancement of photovoltage in graphene," Nature Communications, 2011, 2(1): $1-5$.

182] M. Furchi, A. Urich, A. Pospischil, G. Lilley, K. Unterrainer, H. Detz, et al., "Microcavity-integrated graphene photodetector," Nano Letters, 2012, 12(6): 2773-2777.

[183] I. Nikitskiy, S. Goossens, D. Kufer, T. Lasanta, G. Navickaite, F. H. Koppens, et al., "Integrating an electrically active colloidal quantum dot photodiode with a graphene phototransistor," Nature Communications, 2016, 7(1): 1-8.

[184] S. Y. Chen, Y. Y. Lu, F. Y. Shih, P. H. Ho, Y. F. Chen, C. W. Chen, et al., "Biologically inspired graphene-chlorophyll phototransistors with high gain," Carbon, 2013, 63: 23-29.

[185] G. Konstantatos, M. Badioli, L. Gaudreau, J. Osmond, M. Bernechea, F. P. G. De Arquer, et al., "Hybrid graphene-quantum dot phototransistors with ultrahigh gain," Nature Nanotechnology, 2012, 7(6): 363-368.

[186] P. Afzali, Y. Abdi, and E. Arzi, "Gated graphene/titanium dioxide-based photodetector," Journal of Nanoparticle Research, 2014, 16(10): 2659. 
[187] A. Hu, H. Tian, Q. Liu, L. Wang, L. Wang, X. He, et al., "Graphene on self-assembled InGaN quantum dots enabling ultrahighly sensitive photodetectors," Advanced Optical Materials, 2019, 7(8): 1801792.

[188] C. R. Kagan, E. Lifshitz, E. H. Sargent, and D. V. Talapin, "Building devices from colloidal quantum dots," Science, 2016, 353(6302): aac5523.

[189] C. Giansante, I. Infante, E. Fabiano, R. Grisorio, G. P. Suranna, and G. Gigli, ''Darker-than-Black' $\mathrm{PbS}$ quantum dots: enhancing optical absorption of colloidal semiconductor nanocrystals via short conjugated ligands," Journal of the American Chemical Society, 2015, 137(5): 1875-1886.

[190] W. Guo, S. Xu, Z. Wu, N. Wang, M. Loy, and S. $\mathrm{Du}$, "Oxygen-assisted charge transfer between $\mathrm{ZnO}$ quantum dots and graphene," Small, 2013, 9(18): 3031-3036.

[191] B. Martín-García, A. Polovitsyn, M. Prato, and I. Moreels, "Efficient charge transfer in solution-processed $\mathrm{PbS}$ quantum dot-reduced graphene oxide hybrid materials," Journal of Materials Chemistry C, 2015, 3(27): 7088-7095.

[192] C. W. Chiang, G. Haider, W. C. Tan, Y. R. Liou, Y. C. Lai, R. Ravindranath, et al., "Highly stretchable and sensitive photodetectors based on hybrid graphene and graphene quantum dots," $A C S$ Applied Materials \& Interfaces, 2016, 8(1): 466-471.

[193] S. Darbari, V. Ahmadi, P. Afzali, and Y. Abdi, "Photocatalytic reduction of $\mathrm{GO} / \mathrm{ZnO}$ to achieve GNRs for optoelectronic applications," Journal of Physics D: Applied Physics, 2013, 46(38): 385101.

[194] S. Darbari, V. Ahmadi, P. Afzali, Y. Abdi, and M. Feda, "Reduced graphene oxide/ZnO hybrid structure for high-performance photodetection," Journal of Nanoparticle Research, 2014, 16(12): 2798.

[195] K. B. Ko, B. D. Ryu, M. Han, C. H. Hong, D. A. Dinh, and T. V. Cuong, "Multidimensional graphene and $\mathrm{ZnO}$-based heterostructure for flexible transparent ultraviolet photodetector," Applied Surface Science, 2019, 481: 524-530.

[196] J. E. Muench, A. Ruocco, M. A. Giambra, V. Miseikis, D. Zhang, J. Wang, et al., "Waveguide-integrated, plasmonic enhanced graphene photodetectors," Nano Letters, 2019, 19(11): 7632-7644.

[197] J. Li, C. Zhao, B. Liu, C. You, F. Chu, N. Tian, et al., "Metamaterial grating-integrated graphene photodetector with broadband high responsivity," Applied Surface Science, 2019, 473: 633-640.

[198] B. N. Shivananju, X. Bao, W. Yu, J. Yuan, H. Mu, T. Sun, et al., "Graphene heterostructure integrated optical fiber Bragg grating for light motion tracking and ultrabroadband photodetection from $400 \mathrm{~nm}$ to $10.768 \mu \mathrm{m}$," Advanced Functional
Materials, 2019, 29(19): 1807274

[199] X. Gan, K. F. Mak, Y. Gao, Y. You, F. Hatami, J. Hone, et al., "Strong enhancement of light-matter interaction in graphene coupled to a photonic crystal nanocavity," Nano Letters, 2012, 12(11): 5626-5631.

[200] F. Schedin, E. Lidorikis, A. Lombardo, V. G. Kravets, A. K. Geim, A. N. Grigorenko, et al., "Surface-enhanced Raman spectroscopy of graphene," ACS Nano, 2010, 4(10): 5617-5626.

[201] X. Wang, Z. Cheng, K. Xu, H. K. Tsang, and J. B. Xu, "High-responsivity graphene/siliconheterostructure waveguide photodetectors," Nature Photonics, 2013, 7(11): 888-891.

[202] A. Pospischil, M. Humer, M. M. Furchi, D. Bachmann, R. Guider, T. Fromherz, et al., "CMOS-compatible graphene photodetector covering all optical communication bands," Nature Photonics, 2013, 7(11): 892-896.

[203] S. Su, B. Cheng, C. Xue, W. Wang, Q. Cao, H. Xue, et al., "GeSn pin photodetector for all telecommunication bands detection," Optics Express, 2011, 19(7): 6400-6405.

[204] D. Schall, C. Porschatis, M. Otto, and D. Neumaier, "Graphene photodetectors with a bandwidth larger than $76 \mathrm{GHz}$ fabricated in a 6 inch wafer process line," Journal of Physics D: Applied Physics, 2017, 50(12): 124004.

[205] D. S. Hecht, L. Hu, and G. Irvin, "Emerging transparent electrodes based on thin films of carbon nanotubes, graphene, and metallic nanostructures," Advanced Materials, 2011, 23(13): 1482-1513.

[206]S. De, C. S. Boland, P. J. King, S. Sorel, M. Lotya, U. Patel, et al., "Transparent conducting films from $\mathrm{NbSe}_{3}$ nanowires," Nanotechnology, 2011, 22(28): 285202.

[207] M. B. Gray, D. A. Shaddock, C. C. Harb, and H. A. Bachor, "Photodetector designs for low-noise, broadband, and high-power applications," Review of Scientific Instruments, 1998, 69(11): 3755-3762.

[208] F. Withers, T. H. Bointon, M. F. Craciun, and S. Russo, "All-graphene photodetectors," ACS Nano, 2013, 7(6): 5052-5057.

[209] D. Zhan, L. Sun, Z. H. Ni, L. Liu, X. F. Fan, Y. Wang, et al., " $\mathrm{FeCl}_{3}$-based few-layer graphene intercalation compounds: single linear dispersion electronic band structure and strong charge transfer doping," Advanced Functional Materials, 2010, 20(20): 3504-3509.

[210]S. Sato, H. Ichikawa, N. Iwata, and H. Yamamoto, "Synthesis and characterization of intercalated few-layer graphenes," Japanese Journal of Applied Physics, 2014, 53(2S): 02BD04.

[211] W. Zhao, P. H. Tan, J. Liu, and A. C. Ferrari, "Intercalation of few-layer graphite flakes with 
$\mathrm{FeCl}_{3}$ : Raman determination of Fermi level, layer by layer decoupling, and stability," Journal of the American Chemical Society, 2011, 133(15): 5941-5946.

[212] J. Warmuth, A. Bruix, M. Michiardi, T. Hänke, M. Bianchi, J. Wiebe, et al., "Band-gap engineering by Bi intercalation of graphene on Ir (111)," Physical Review B, 2016, 93(16): 165437.

[213] I. Khrapach, F. Withers, T. H. Bointon, D. K. Polyushkin, W. L. Barnes, S. Russo, et al., "Novel highly conductive and transparent graphene-based conductors," Advanced Materials, 2012, 24(21): 2844-2849.

[214] A. De Sanctis, G. F. Jones, D. J. Wehenkel, F. Bezares, F. H. Koppens, M. F. Craciun, et al., "Extraordinary linear dynamic range in laser-defined functionalized graphene photodetectors," Science Advances, 2017, 3(5): e1602617.

[215] S. Wang, Y. Sekine, S. Suzuki, F. Maeda, and H. Hibino, "Photocurrent generation of a single-gate graphene $\mathrm{p}-\mathrm{n}$ junction fabricated by interfacial modification," Nanotechnology, 2015, 26(38): 385203.

[216] M. Kim, S. M. Choi, H. A. Yoon, S. K. Choi, J. U. Lee, J. Kim, et al., "Photocurrent generation at $\mathrm{ABA} / \mathrm{ABC}$ lateral junction in tri-layer graphene photodetector," Carbon, 2016, 96: 454-458.

[217] C. Cong, T. Yu, K. Sato, J. Shang, R. Saito, G. F. Dresselhaus, et al., "Raman characterization of ABA-and ABC-stacked trilayer graphene," ACS Nano, 2011, 5(11): 8760-8768.

[218] S. H. Cheng, T. M. Weng, M. L. Lu, W. C. Tan, J. Y. Chen, and Y. F. Chen, "All carbon-based photodetectors: an eminent integration of graphite quantum dots and two dimensional graphene," Scientific Reports, 2013, 3: 2694.

[219] V. Gupta, N. Chaudhary, R. Srivastava, G. D. Sharma, R. Bhardwaj, and S. Chand, "Luminscent graphene quantum dots for organic photovoltaic devices," Journal of the American Chemical Society, 2011, 133(26): 9960-9963.

[220] Y. Li, Y. Hu, Y. Zhao, G. Shi, L. Deng, Y. Hou, et al., "An electrochemical avenue to green-luminescent graphene quantum dots as potential electron-acceptors for photovoltaics," Advanced Materials, 2011, 23(6): 776-780.

[221] S. Zhu, J. Zhang, X. Liu, B. Li, X. Wang, S. Tang, et al., "Graphene quantum dots with controllable surface oxidation, tunable fluorescence and up-conversion emission," Rsc Advances, 2012, 2(7): 2717-2720.

[222] V. C. Tung, L.-M. Chen, M. J. Allen, J. K. Wassei, K. Nelson, R. B. Kaner, et al., "Low-temperature solution processing of graphene-carbon nanotube hybrid materials for high-performance transparent conductors," Nano Letters, 2009, 9(5): 1949-1955.

[223] P. J. King, U. Khan, M. Lotya, S. De, and J. N. Coleman, "Improvement of transparent conducting nanotube films by addition of small quantities of graphene," ACS Nano, 2010, 4(7): 4238-4246.

[224] X. Yu, Z. Dong, J. K. Yang, and Q. J. Wang, "Room-temperature mid-infrared photodetector in all-carbon graphene nanoribbon- $\mathrm{C}_{60}$ hybrid nanostructure," Optica, 2016, 3(9): 979-984.

[225] Z. Sun, Z. Liu, J. Li, G. Tai, S. P. Lau, and F. Yan, "Infrared photodetectors based on CVD-grown graphene and $\mathrm{PbS}$ quantum dots with ultrahigh responsivity," Advanced Materials, 2012, 24(43): 5878-5883.

[226] D. Zhang, L. Gan, Y. Cao, Q. Wang, L. Qi, and $X$. Guo, "Understanding charge transfer at PbS-decorated graphene surfaces toward a tunable photosensor," Advanced Materials, 2012, 24(20): 2715-2720.

[227] S. M. Song, J. K. Park, O. J. Sul, and B. J. Cho, "Determination of work function of graphene under a metal electrode and its role in contact resistance," Nano Letters, 2012, 12(8): 3887-3892.

[228] J. G. Son, M. Son, K. J. Moon, B. H. Lee, J. M. Myoung, M. S. Strano, et al., "Sub-10 nm graphene nanoribbon array field-effect transistors fabricated by block copolymer lithography," Advanced Materials, 2013, 25(34): 4723-4728.

[229]Y. Zhang, T. Liu, B. Meng, X. Li, G. Liang, X. Hu, et al., "Broadband high photoresponse from pure monolayer graphene photodetector," Nature Communications, 2013, 4(1): 1811.

[230] S. Du, W. Lu, A. Ali, P. Zhao, K. Shehzad, H. Guo, et al., "A broadband fluorographene photodetector," Advanced Materials, 2017, 29(22): 1700463.

[231] O. Leenaerts, H. Peelaers, A. Hernández-Nieves, B. Partoens, and F. Peeters, "First-principles investigation of graphene fluoride and graphane," Physical Review B, 2010, 82(19): 195436. 